\title{
Stereoselective Synthesis of the Decahydroquinoline Alkaloid cis-195J
}

\author{
Rudina Veliu and Christoph Schneider* \\ Institut für Organische Chemie, Universität Leipzig, Johannisallee 29, D-04103 Leipzig, Germany \\ Email: schneider@chemie@.uni-leipzig.de.
}

\section{Table of Contents:}

Spectral data ( ${ }^{1} \mathrm{H}$ NMR and ${ }^{13} \mathrm{C}$ NMR) of the new compounds

S2-S20

1D NOESY spectra of $\mathbf{2 2 a}$ and $\mathbf{2 2} \mathbf{b}$

S21-S22

HPLC Chromatogram of compound 7

S23

Table with the NMR data of $c i s-195 \mathrm{~J}-\mathbf{a}$ and cis-195J-b

S24 
(⿸丆口

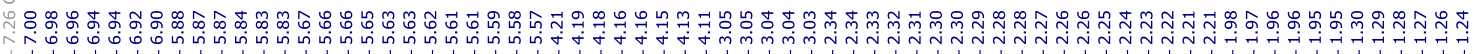

23

${ }^{1} \mathrm{H} \mathrm{NMR}\left(\mathrm{CDCl}_{3}, 400 \mathrm{MHz}\right)$
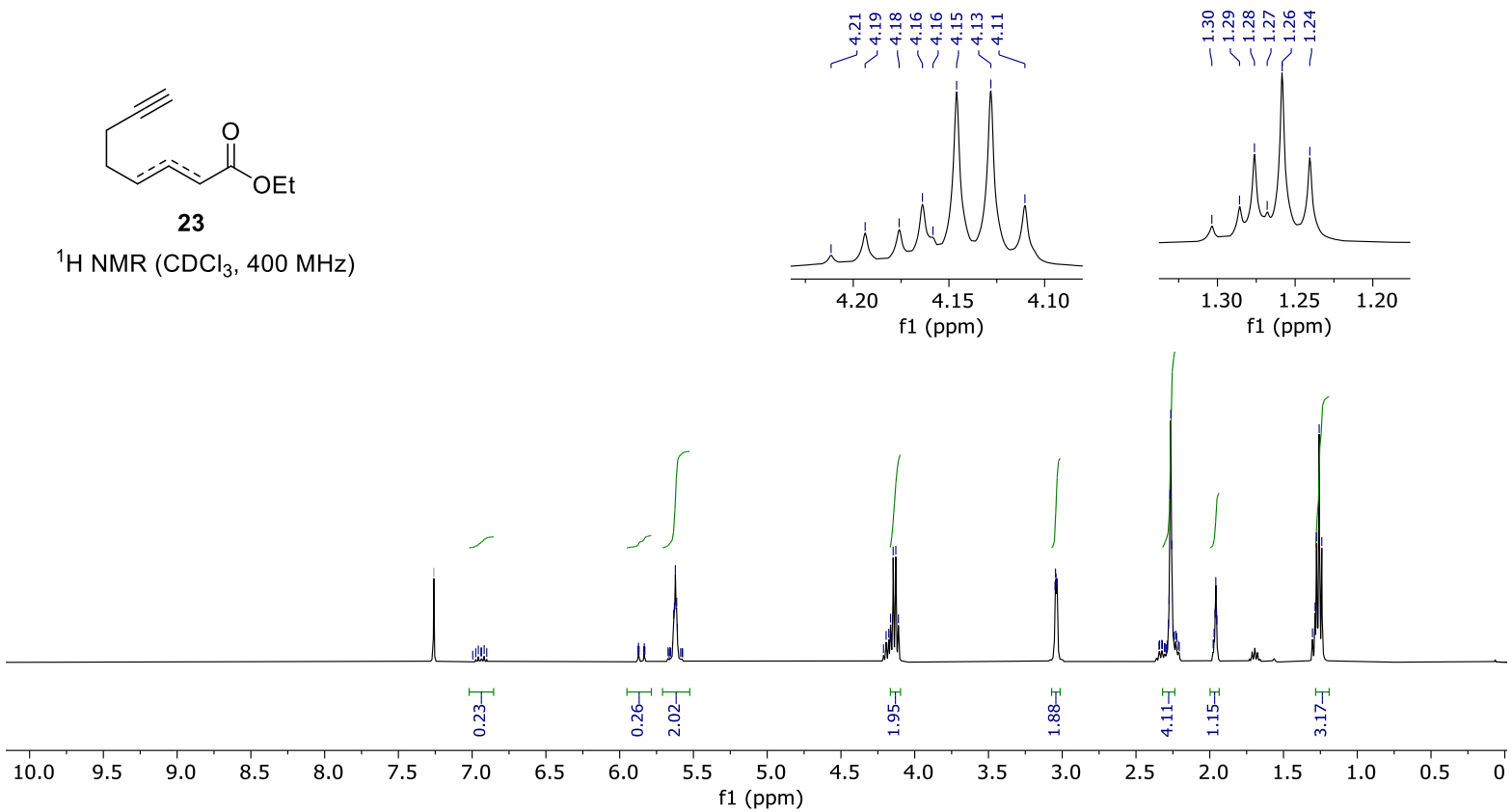

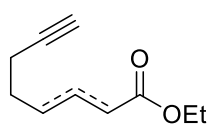

23

${ }^{13} \mathrm{C}\left\{{ }^{1} \mathrm{H}\right\}$ NMR $\left(\mathrm{CDCl}_{3}, 100 \mathrm{MHz}\right)$
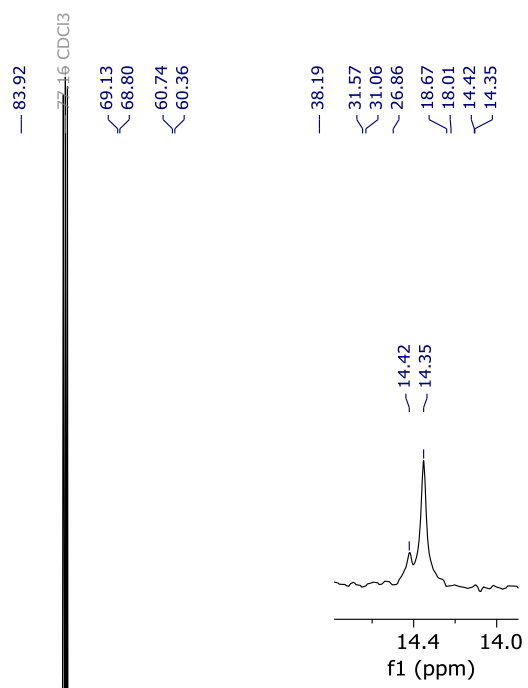

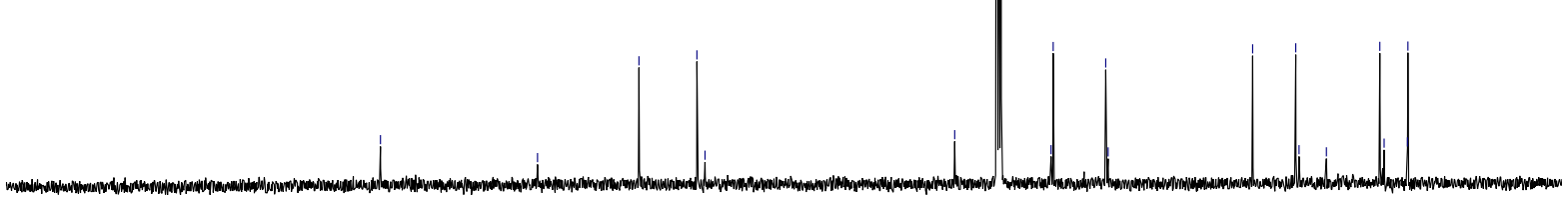

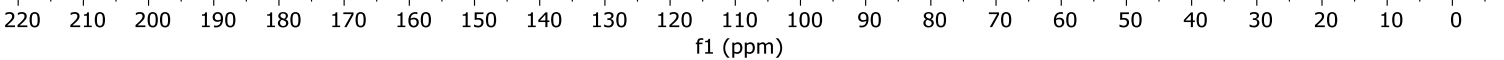




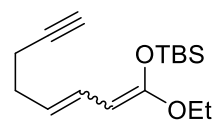

${ }^{1} \mathrm{H} \mathrm{NMR}\left(\mathrm{CDCl}_{3}, 400 \mathrm{MHz}\right)$

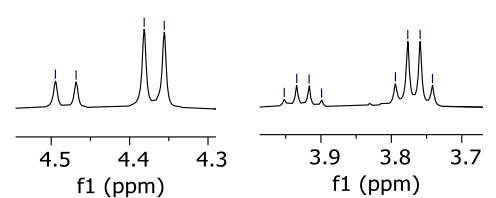

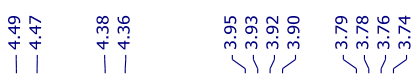

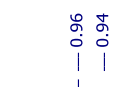

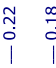

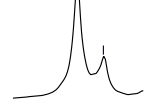

$\begin{array}{lll}0.96 & 0.93\end{array}$

f1 (ppm)

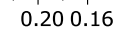

f1 (ppm)

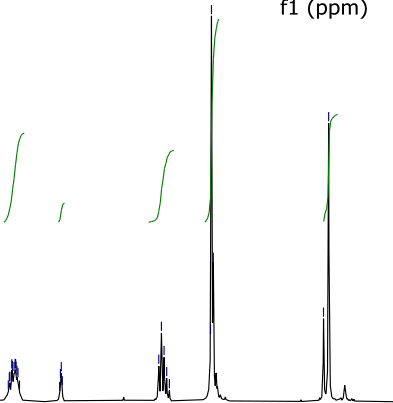

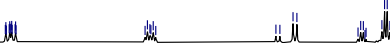

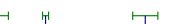

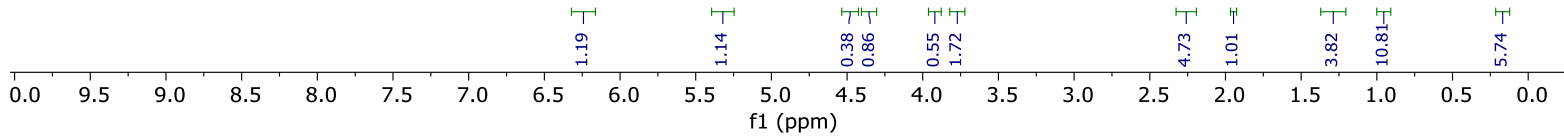

li

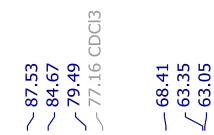

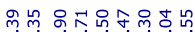

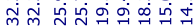

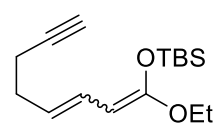

6

${ }^{13} \mathrm{C}\left\{{ }^{1} \mathrm{H}\right\}$ NMR $\left(\mathrm{CDCl}_{3}, 101 \mathrm{MHz}\right)$

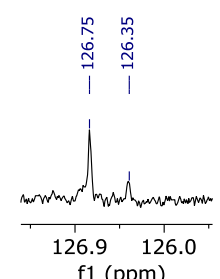

f1 (ppm)
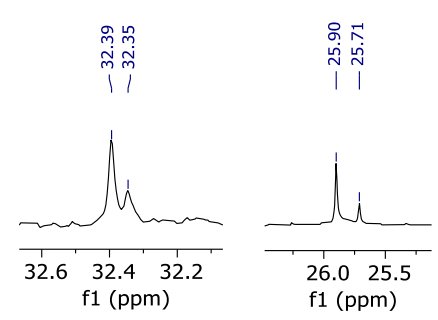


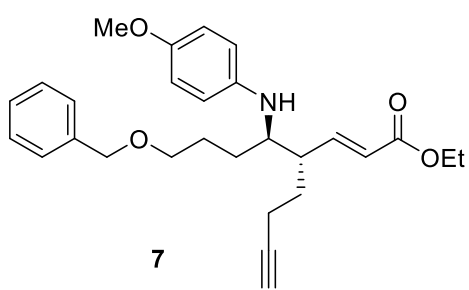

${ }^{1} \mathrm{H} \mathrm{NMR}\left(\mathrm{CDCl}_{3}, 300 \mathrm{MHz}\right)$

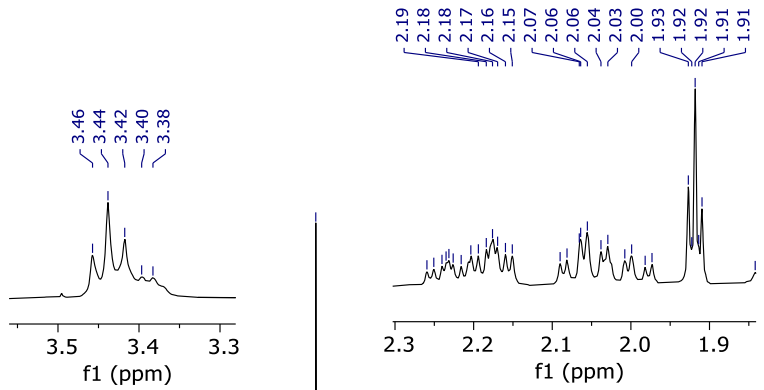

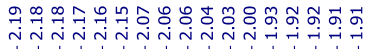

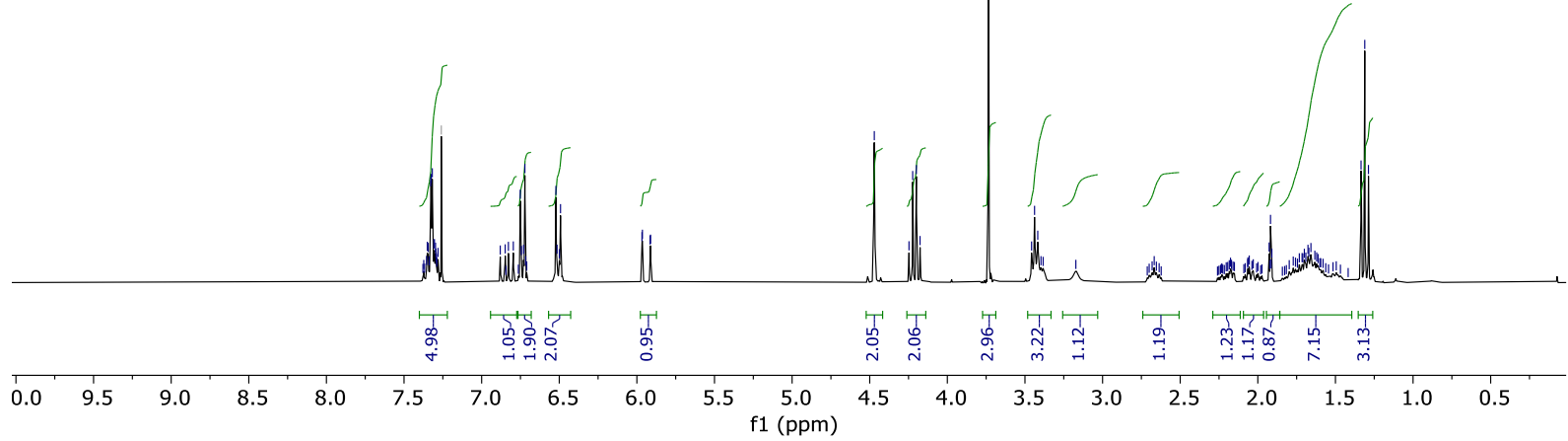

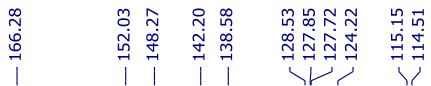
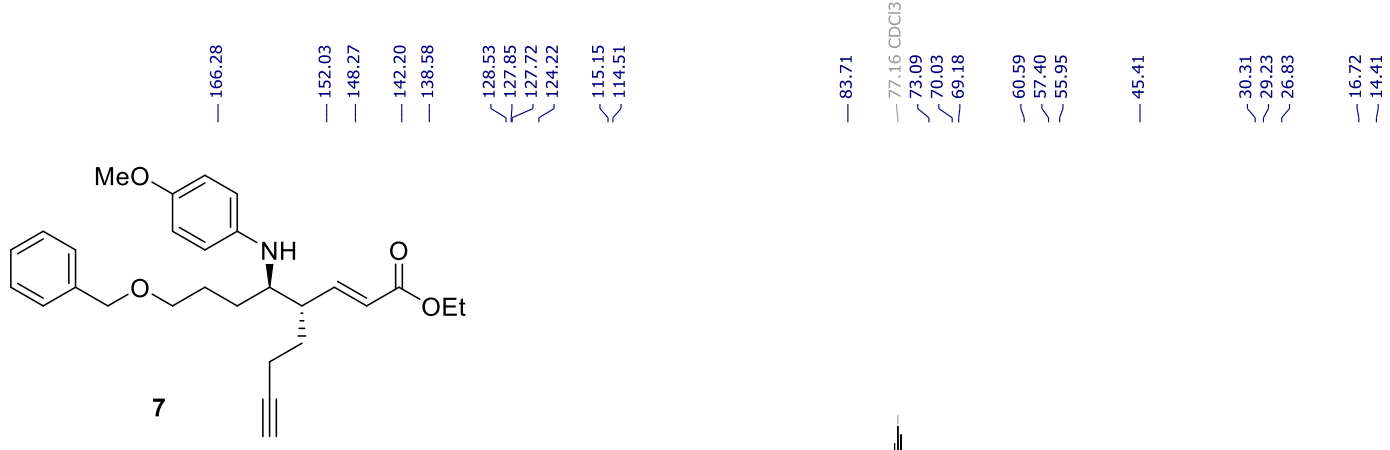

${ }^{13} \mathrm{C}\left\{{ }^{1} \mathrm{H}\right\}$ (APT) NMR $\left(\mathrm{CDCl}_{3}, 101 \mathrm{MHz}\right)$

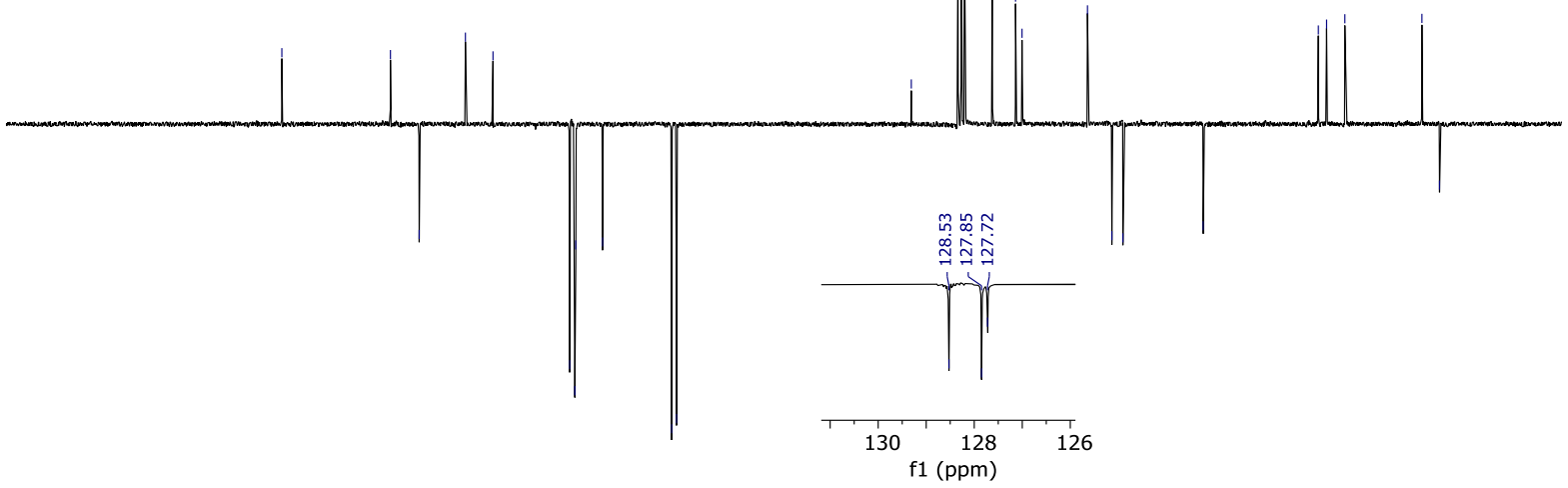

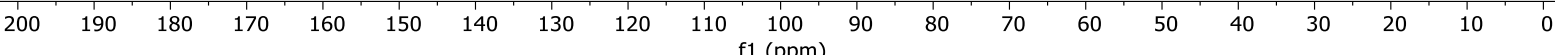




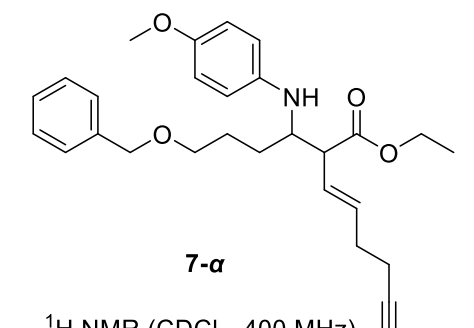

${ }^{1} \mathrm{H} \mathrm{NMR}\left(\mathrm{CDCl}_{3}, 400 \mathrm{MHz}\right) \quad \|$
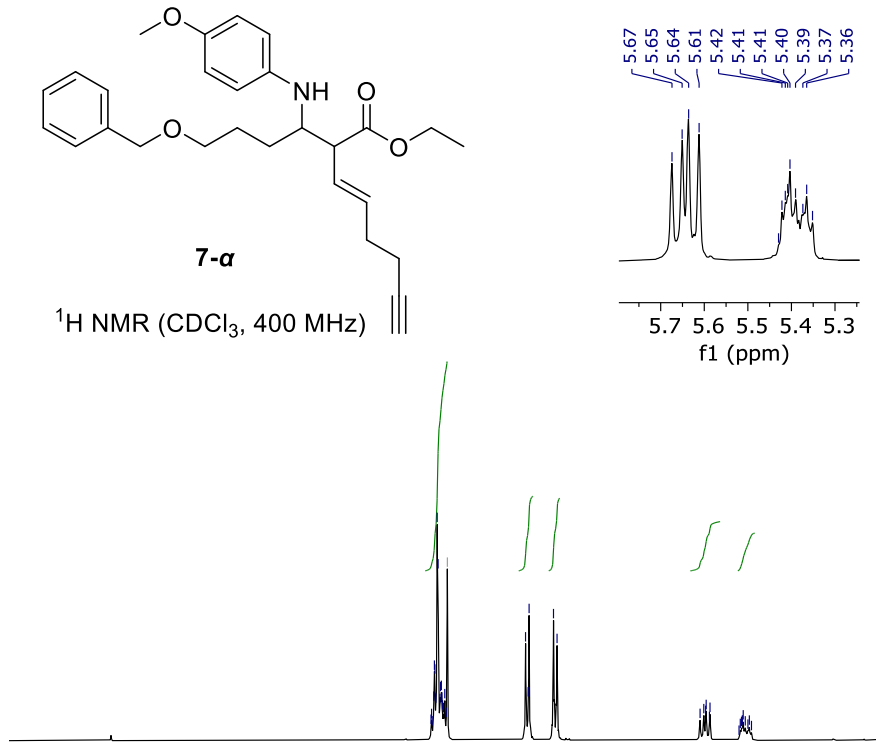
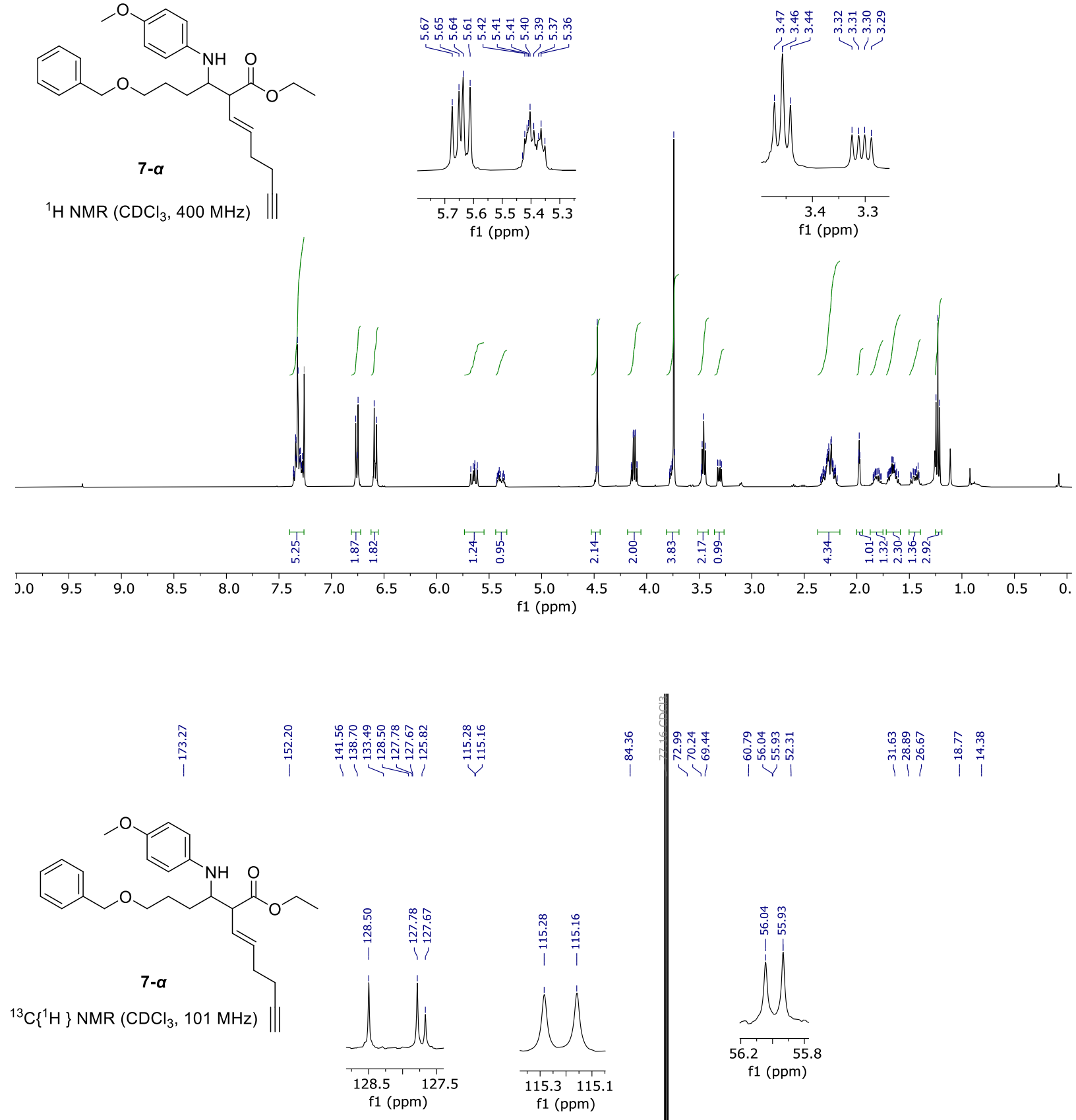

f1 (ppm)

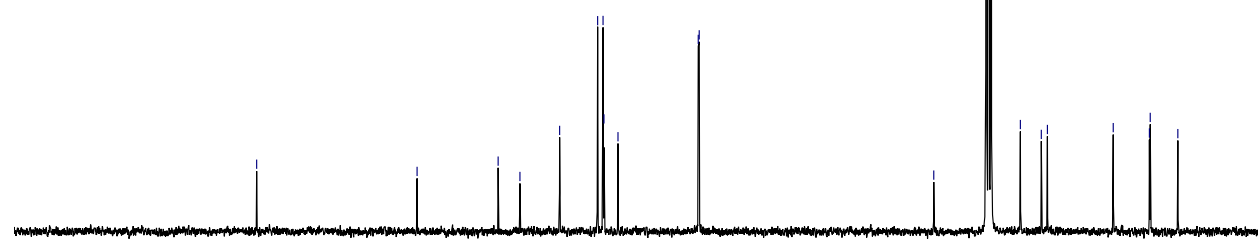

200

1,1

100
$\mathrm{f} 1(\mathrm{ppm})$ 


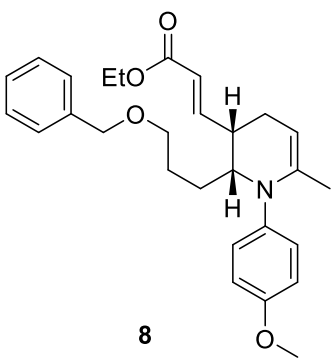

${ }^{1} \mathrm{H} \mathrm{NMR}\left(\mathrm{CDCl}_{3}, 400 \mathrm{MHz}\right)$

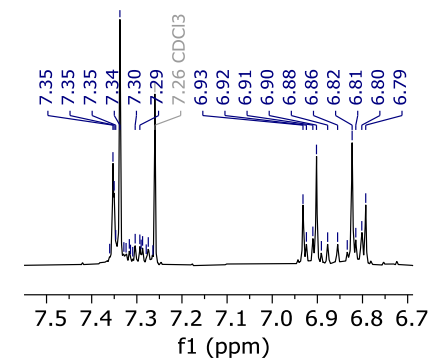

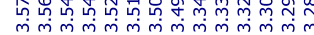

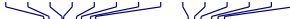

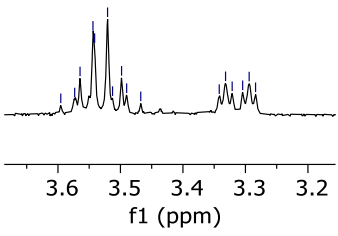

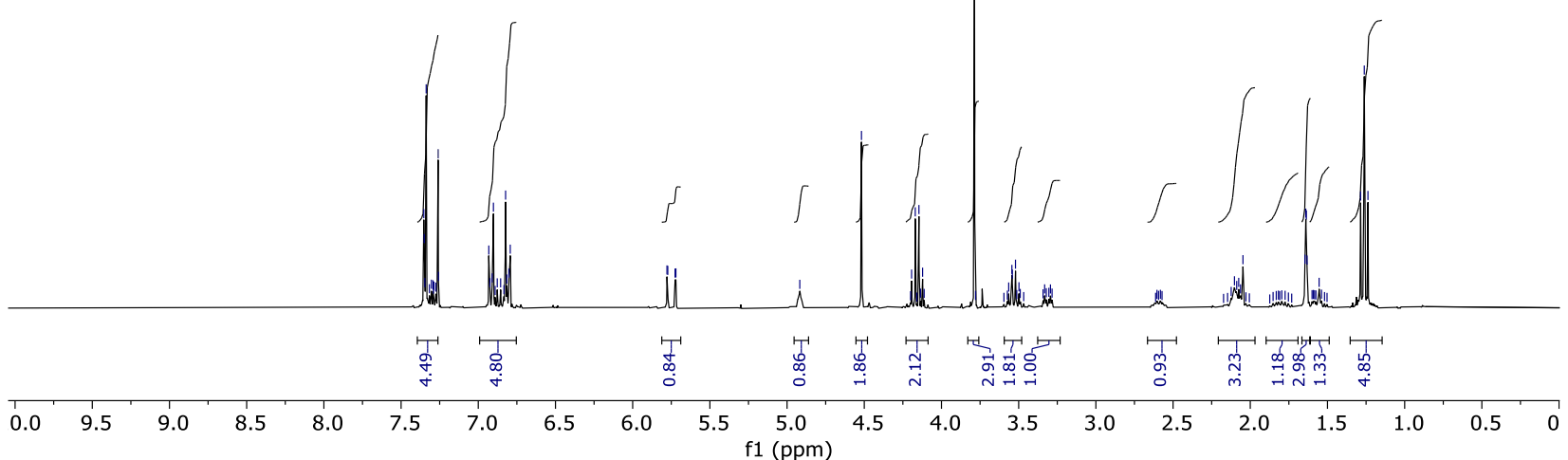

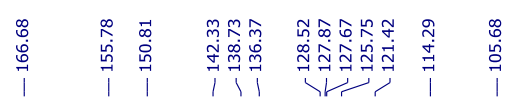

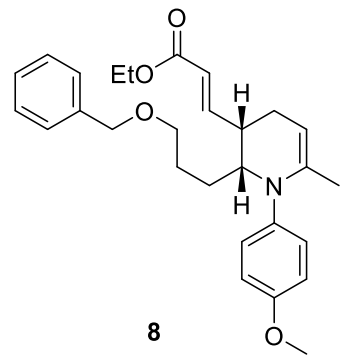

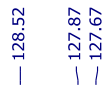

${ }^{13} \mathrm{C}\left\{{ }^{1} \mathrm{H}\right\}$ NMR $\left(\mathrm{CDCl}_{3}, 101 \mathrm{MHz}\right)$
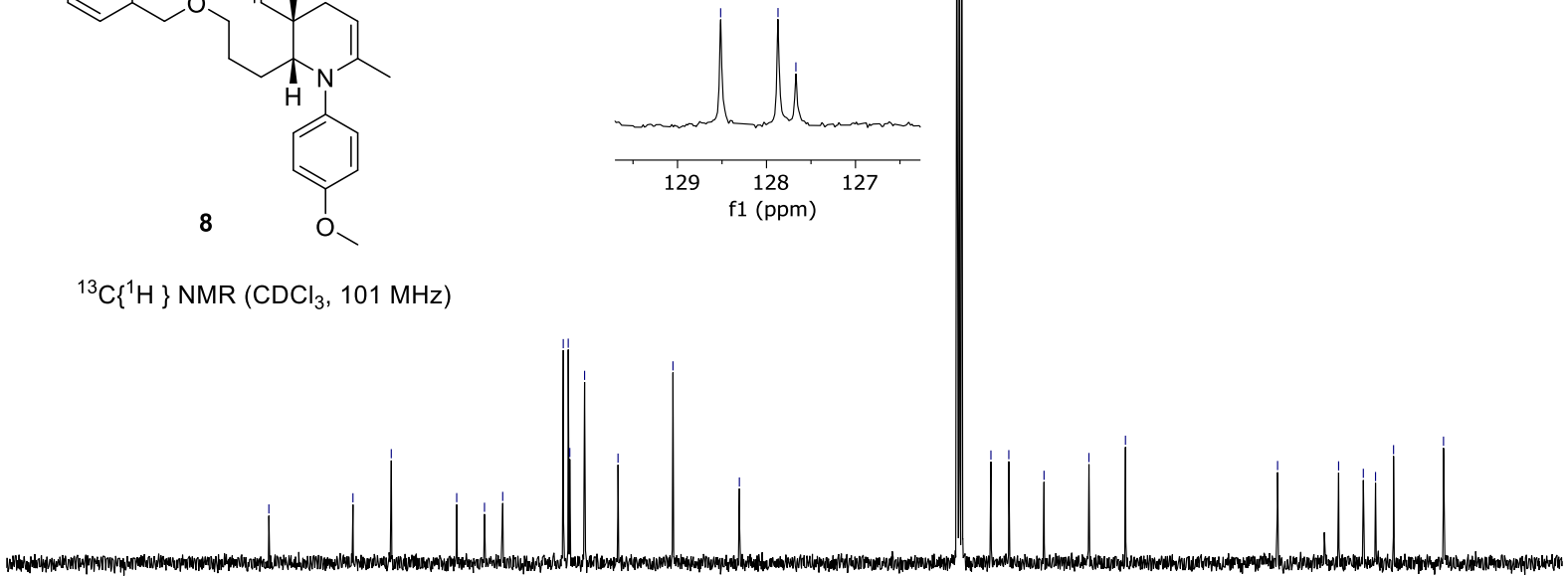

$: 00$

$\begin{array}{llllll}90 & 180 & 170 & 160 & 150 & 140\end{array}$

$\begin{array}{lll}130 & 120 & 110\end{array}$ 100
$\mathrm{f} 1(\mathrm{ppm})$ 


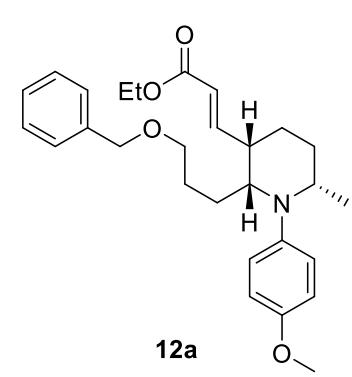

${ }^{1} \mathrm{H} \mathrm{NMR}\left(\mathrm{CDCl}_{3}, 400 \mathrm{MHz}\right)$

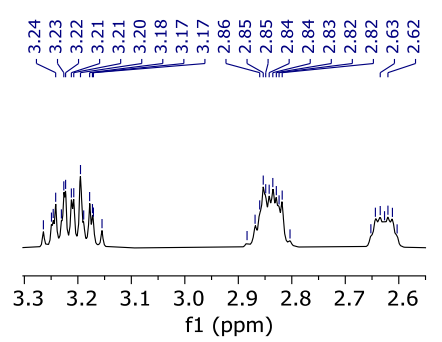

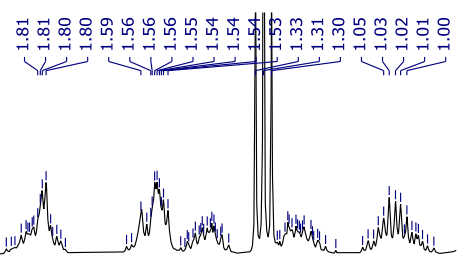

\begin{tabular}{llllllllllllllll}
\hline & 1.9 & 1.8 & 1.7 & 1.6 & 1.5 & 1.4 & 1.3 & 1.2 & 1.1 & 1.0 & 0.9
\end{tabular}

f1 (ppm)

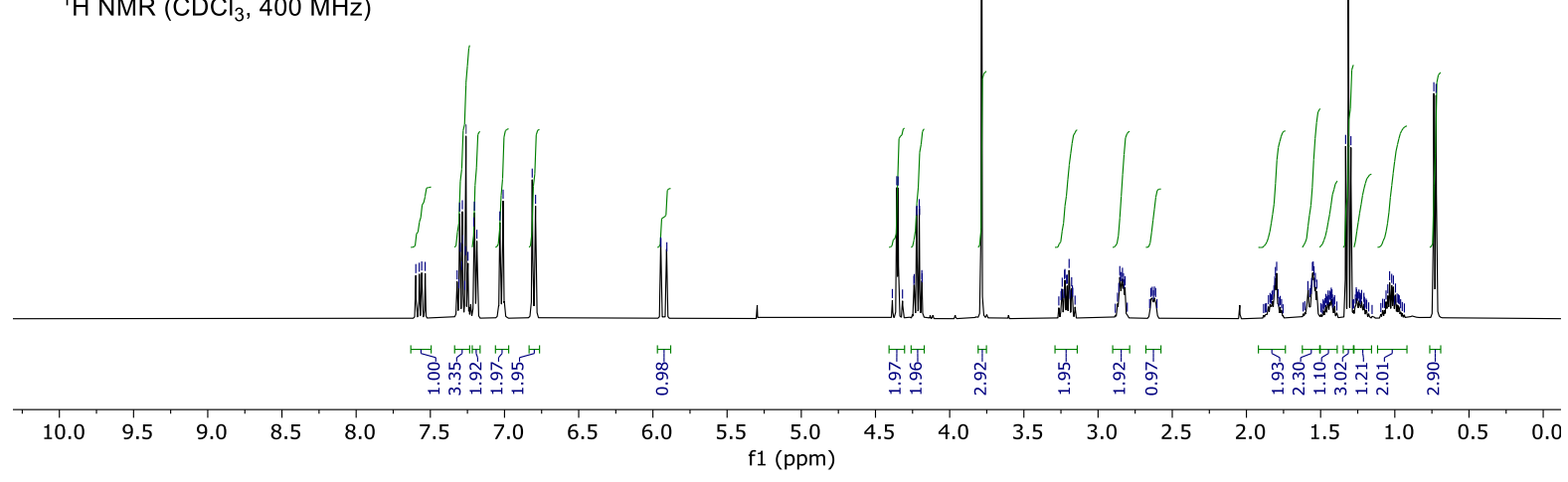

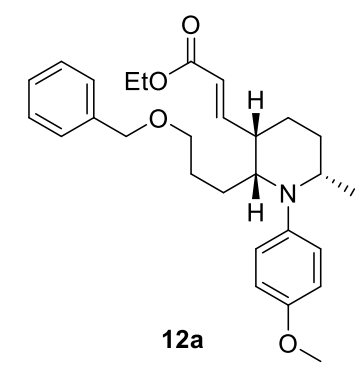

${ }^{13} \mathrm{C}\left\{{ }^{1} \mathrm{H}\right\}$ NMR $\left(\mathrm{CDCl}_{3}, 101 \mathrm{MHz}\right)$

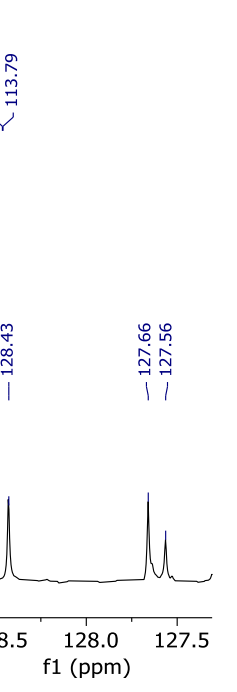

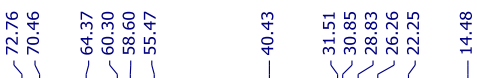

(1)

$\begin{array}{lllll}114.5 & 114.0 & 113.5 & 113.0\end{array}$

f1 (ppm)

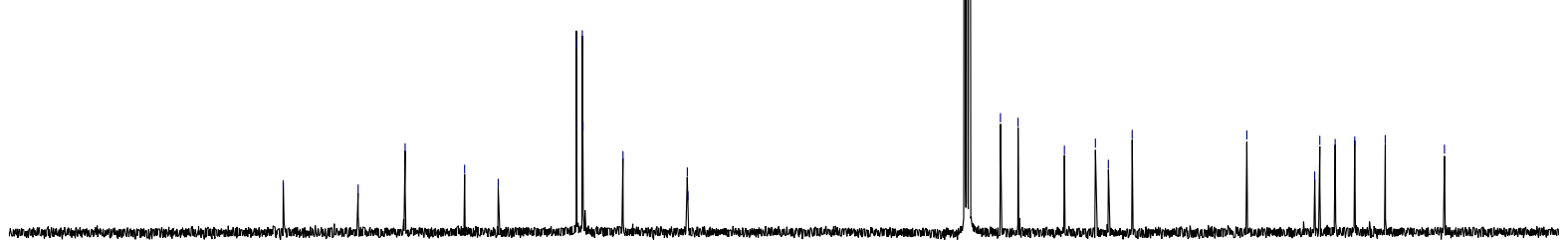



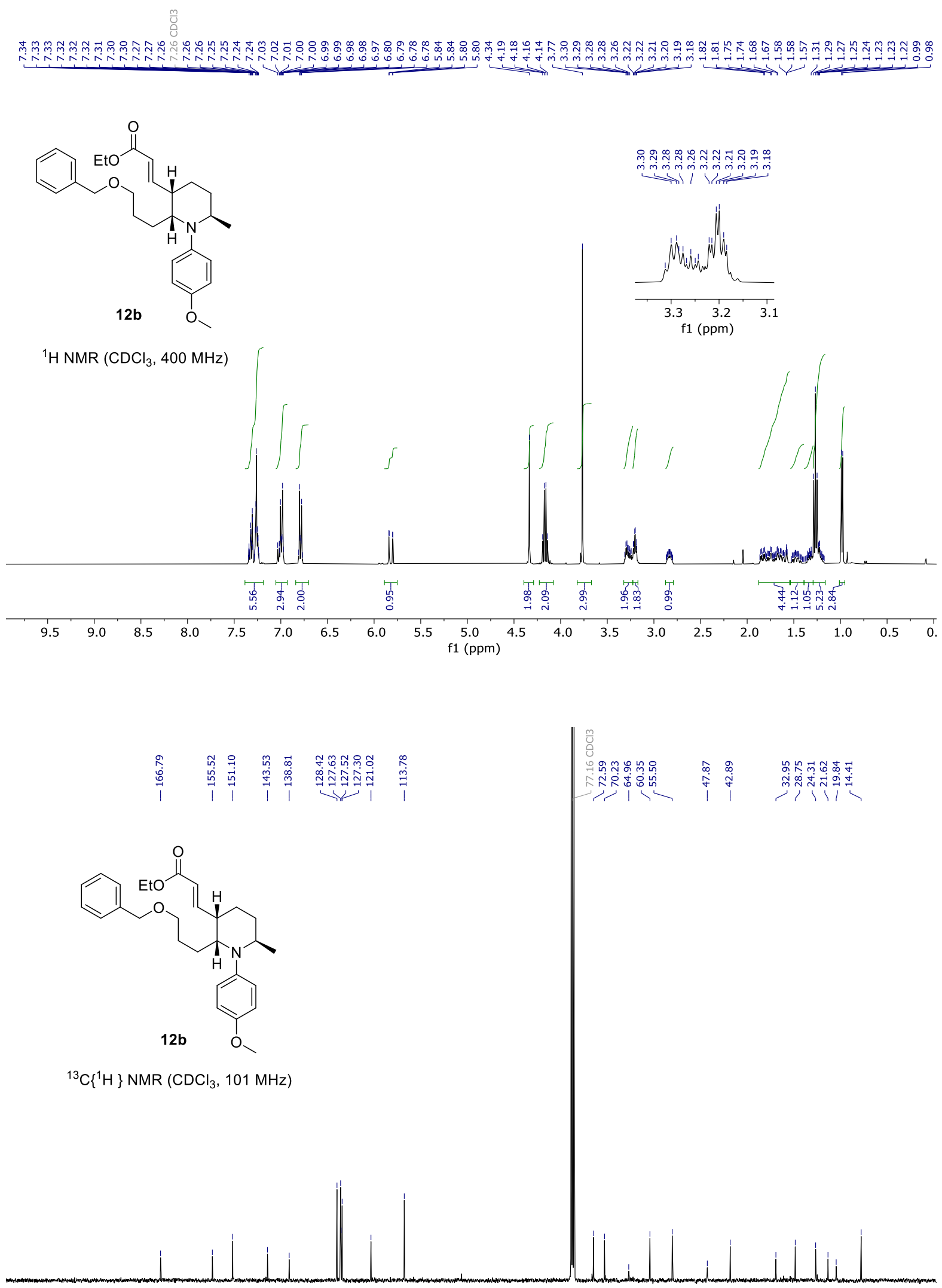

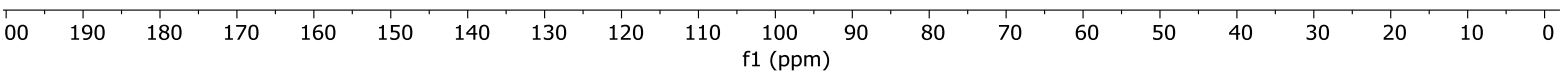




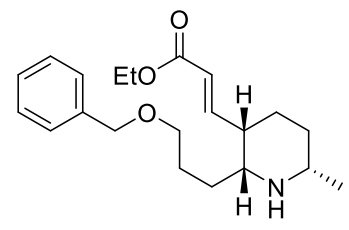

14

${ }^{1} \mathrm{H} \mathrm{NMR}\left(\mathrm{CDCl}_{3}, 400 \mathrm{MHz}\right)$

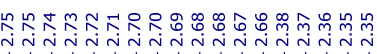

(n)

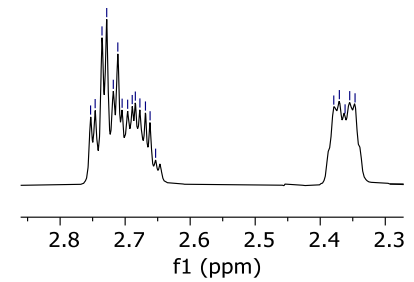

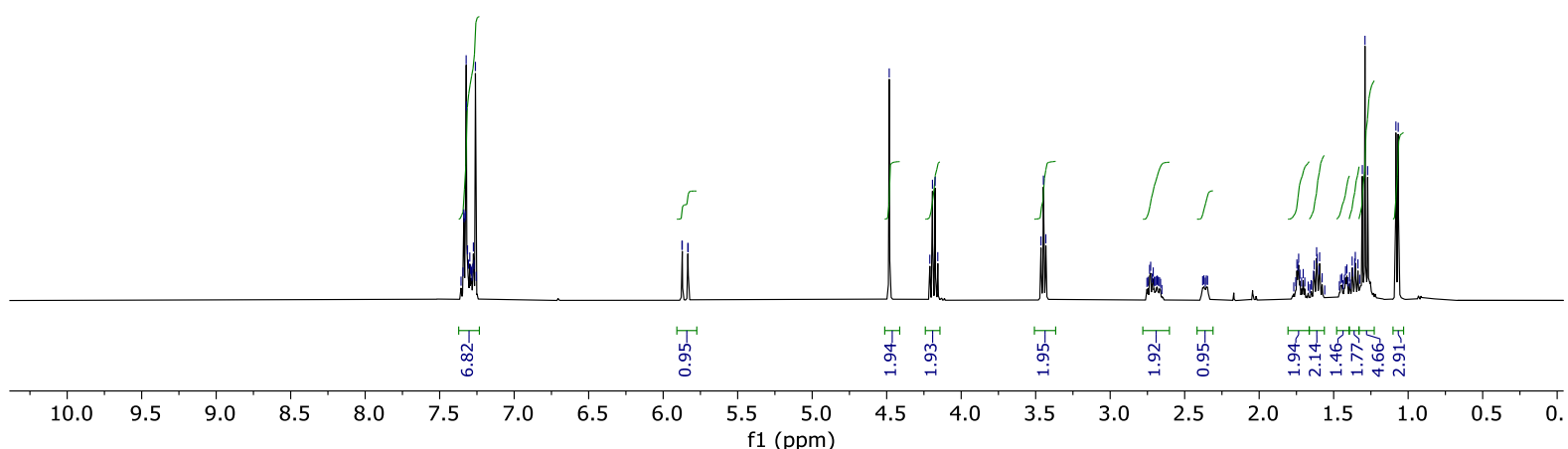

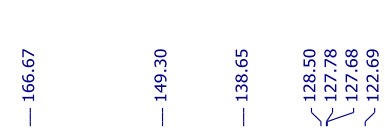<smiles>CCOC(=O)/C=C/[C@H]1CC[C@@H](C)N[C@H]1CCCOCc1ccccc1</smiles>

14

${ }^{13} \mathrm{C}\left\{{ }^{1} \mathrm{H}\right\}$ (APT) NMR $\left(\mathrm{CDCl}_{3}, 101 \mathrm{MHz}\right)$

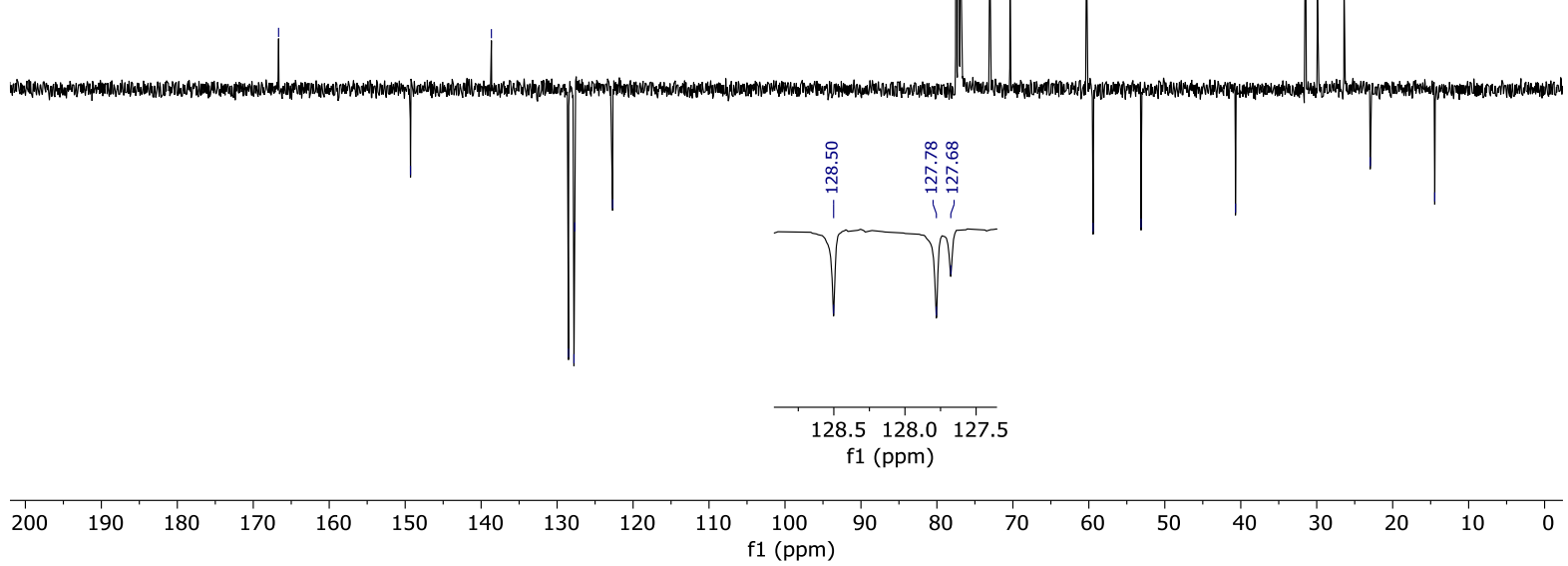



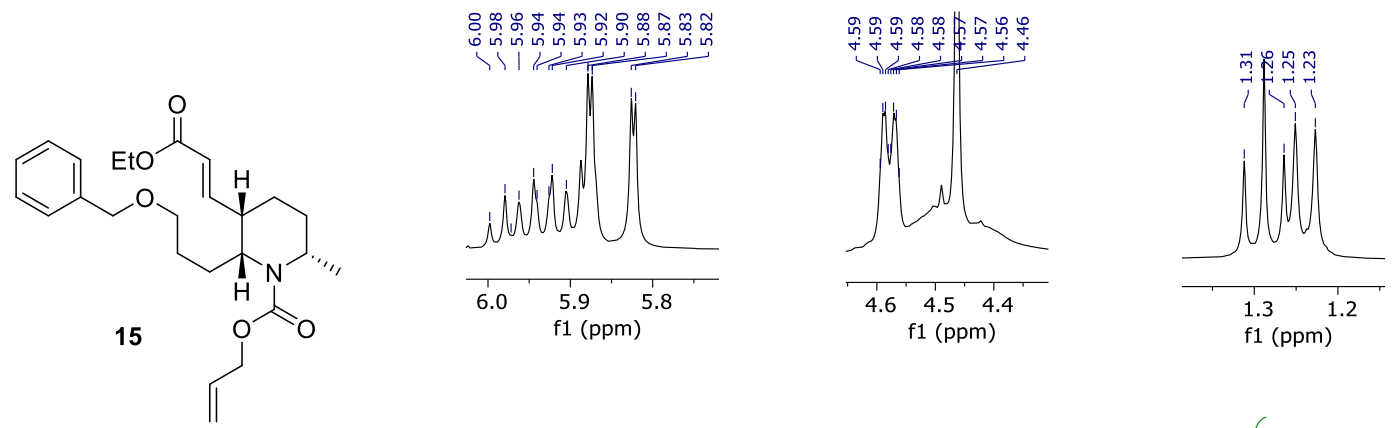

${ }^{1} \mathrm{H} \mathrm{NMR}\left(\mathrm{CDCl}_{3}, 400 \mathrm{MHz}\right)$

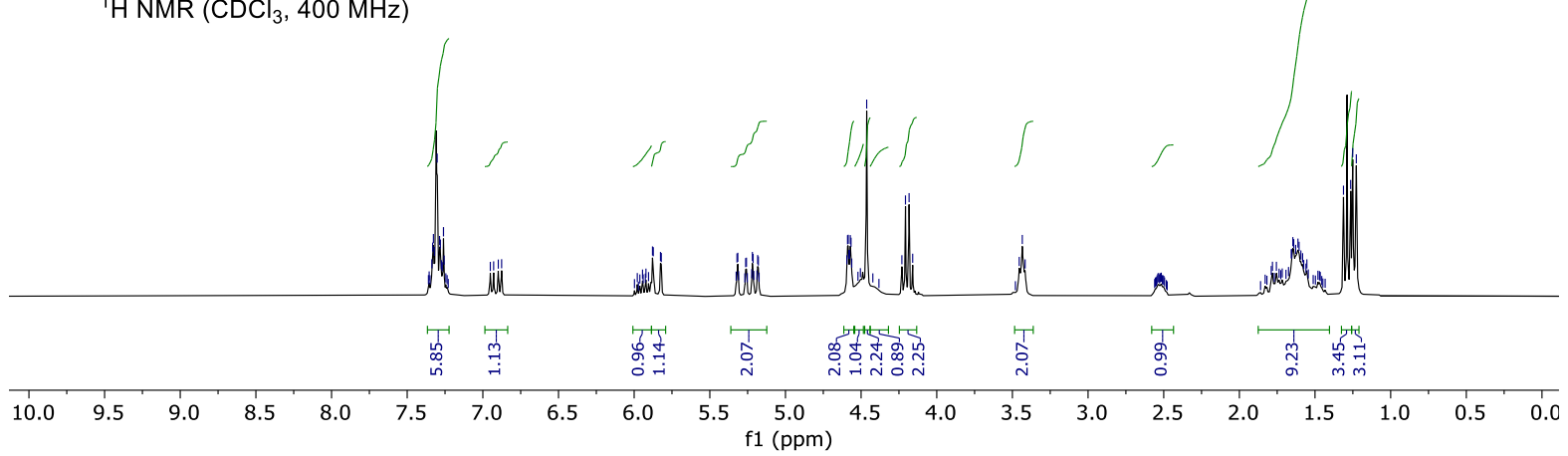

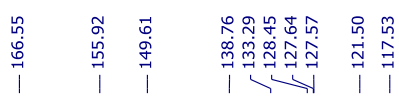
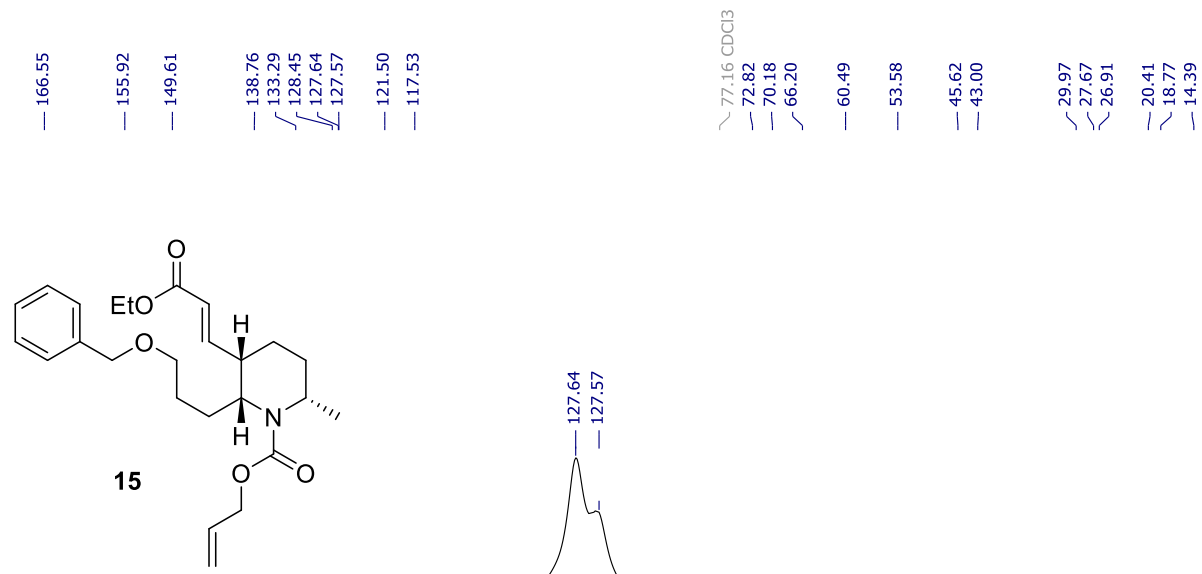

${ }^{13} \mathrm{C}\left\{{ }^{1} \mathrm{H}\right\} \operatorname{NMR}\left(\mathrm{CDCl}_{3}, 101 \mathrm{MHz}\right)$
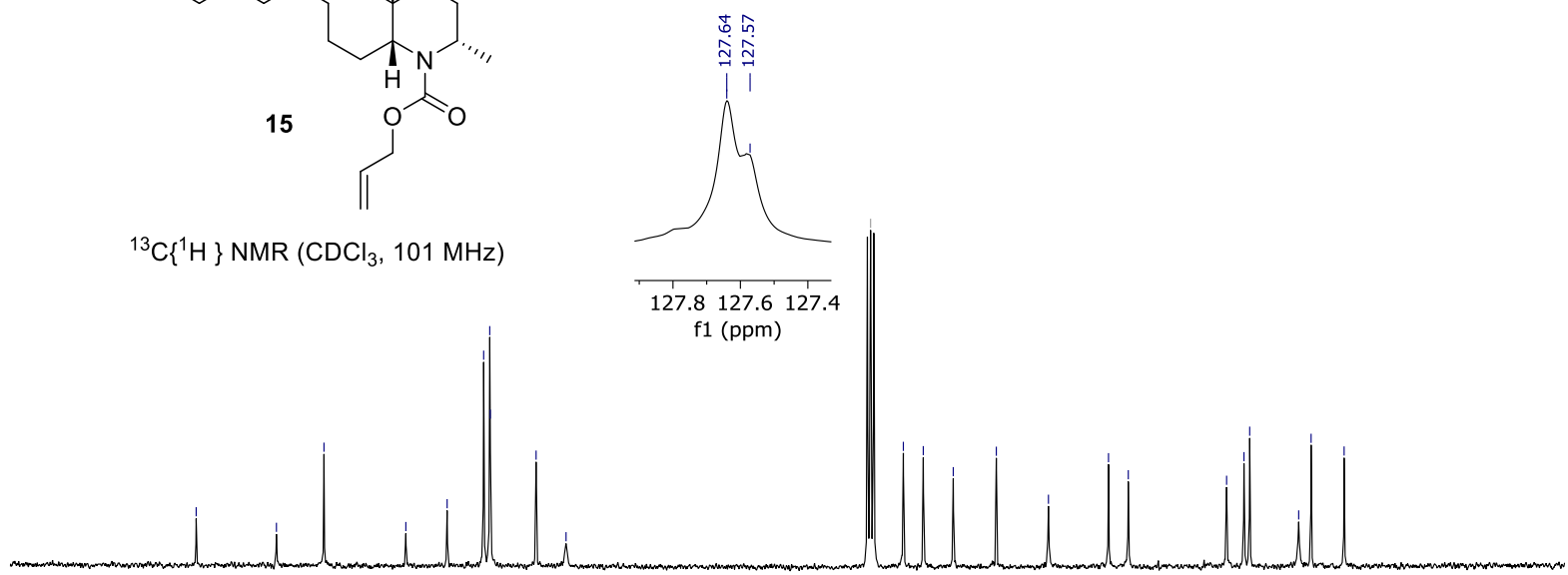

$\begin{array}{llllllllllllllllllllll}190 & 180 & 170 & 160 & 150 & 140 & 130 & 120 & 110 & 100 & \begin{array}{c}90 \\ \mathrm{f} 1(\mathrm{ppm})\end{array} & 80 & 70 & 60 & 50 & 40 & 30 & 20 & 10 & 0 & -10\end{array}$ 


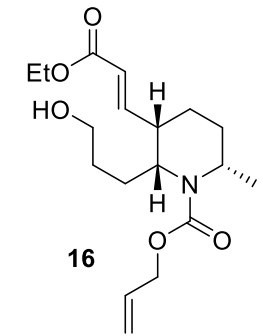

${ }^{1} \mathrm{H} \mathrm{NMR}\left(\mathrm{CDCl}_{3}, 400 \mathrm{MHz}\right)$
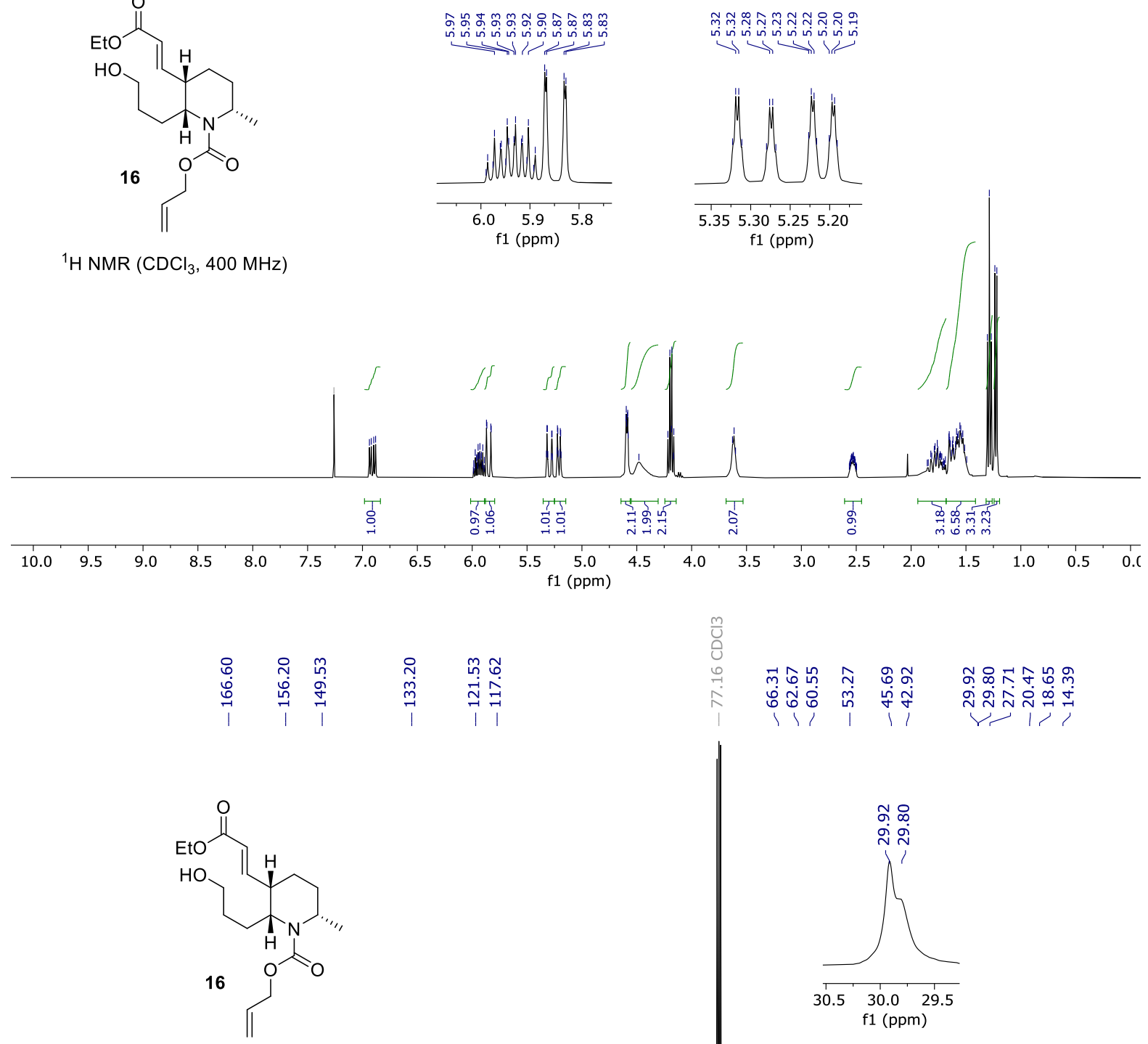

${ }^{13} \mathrm{C}\left\{{ }^{1} \mathrm{H}\right\} \mathrm{NMR}\left(\mathrm{CDCl}_{3}, 101 \mathrm{MHz}\right)$
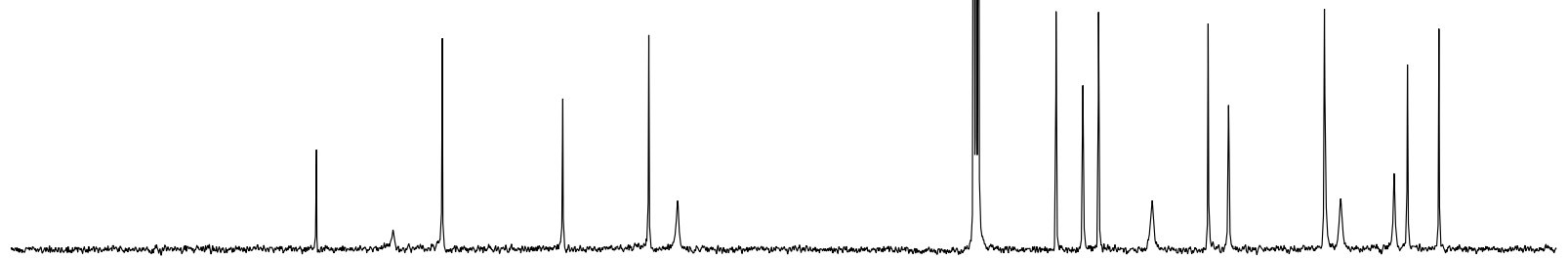

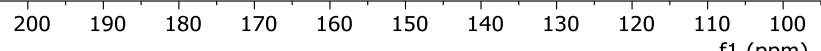

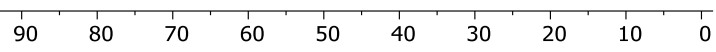



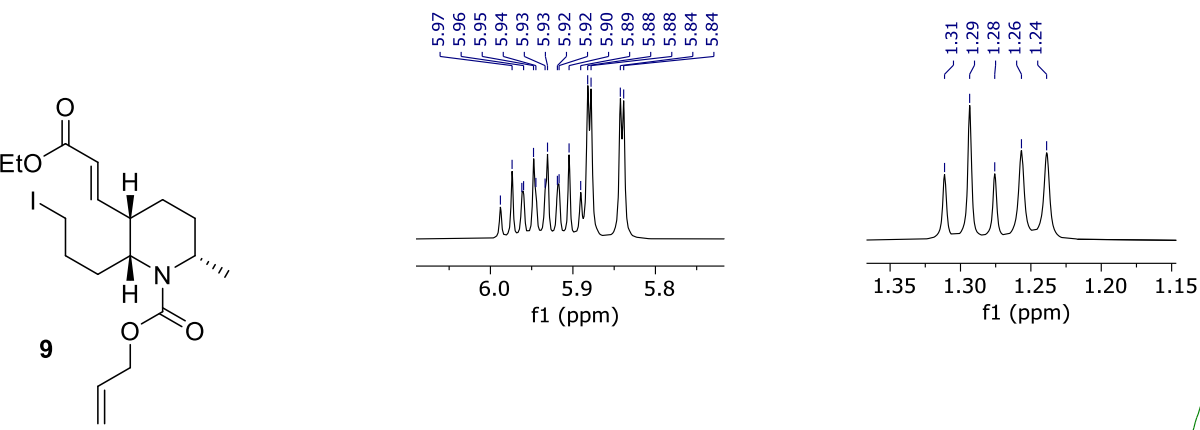

${ }^{1} \mathrm{H} \mathrm{NMR}\left(\mathrm{CDCl}_{3}, 400 \mathrm{MHz}\right)$

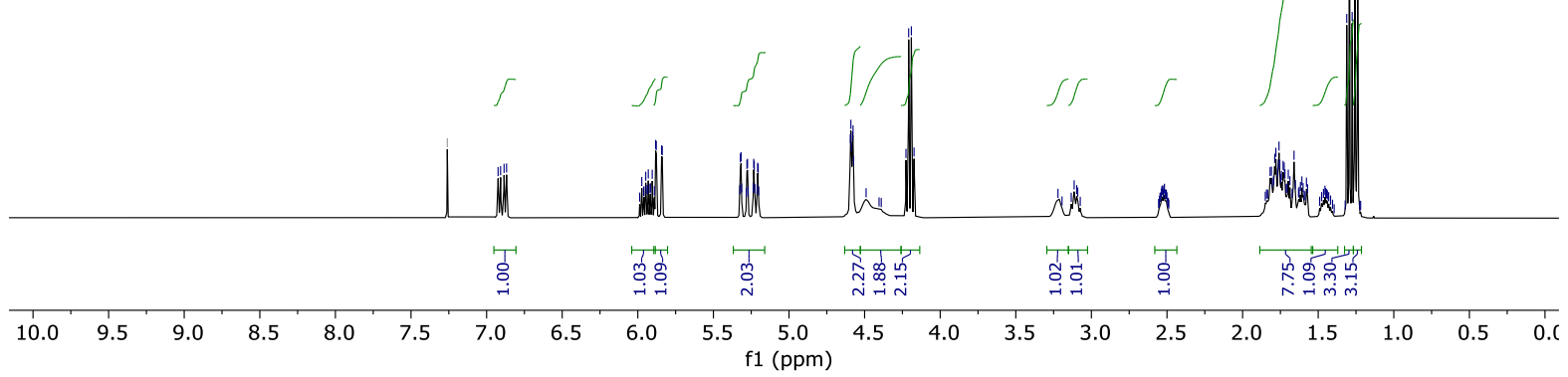

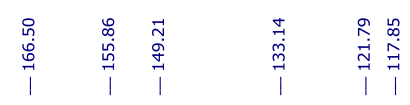
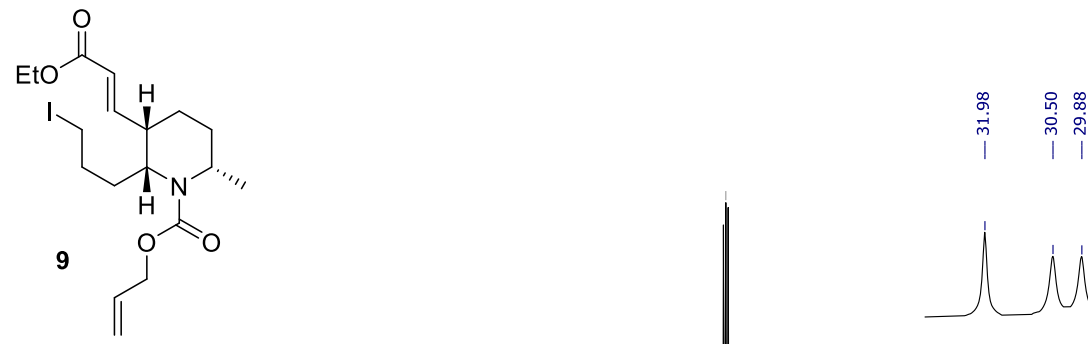

${ }^{13} \mathrm{C}\left\{{ }^{1} \mathrm{H}\right\} \mathrm{NMR}\left(\mathrm{CDCl}_{3}, 101 \mathrm{MHz}\right)$

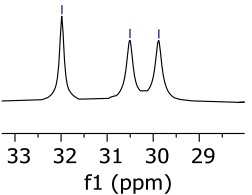

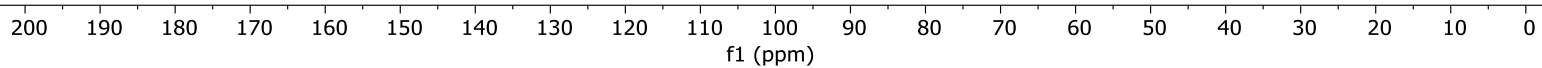




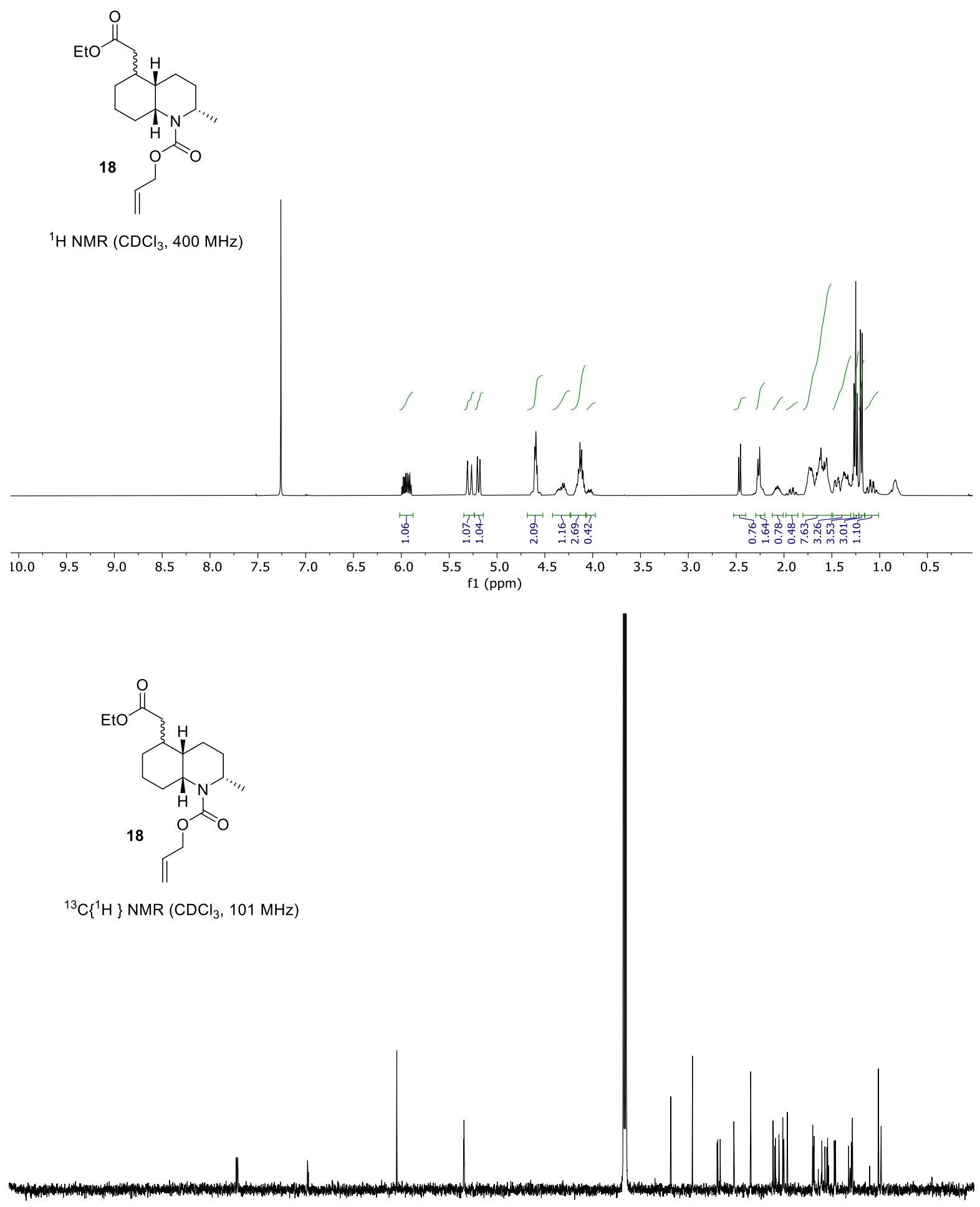

$\begin{array}{llllllllllllllllllllllllllll}220 & 210 & 200 & 190 & 180 & 170 & 160 & 150 & 140 & 130 & 120 & 110 & 100 & 90 & 80 & 70 & 60 & 50 & 40 & 30 & 20 & 10 & 0 & \end{array}$ 


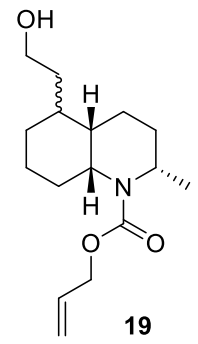

${ }^{1} \mathrm{H} \mathrm{NMR}\left(\mathrm{CDCl}_{3}, 400 \mathrm{MHz}\right)$
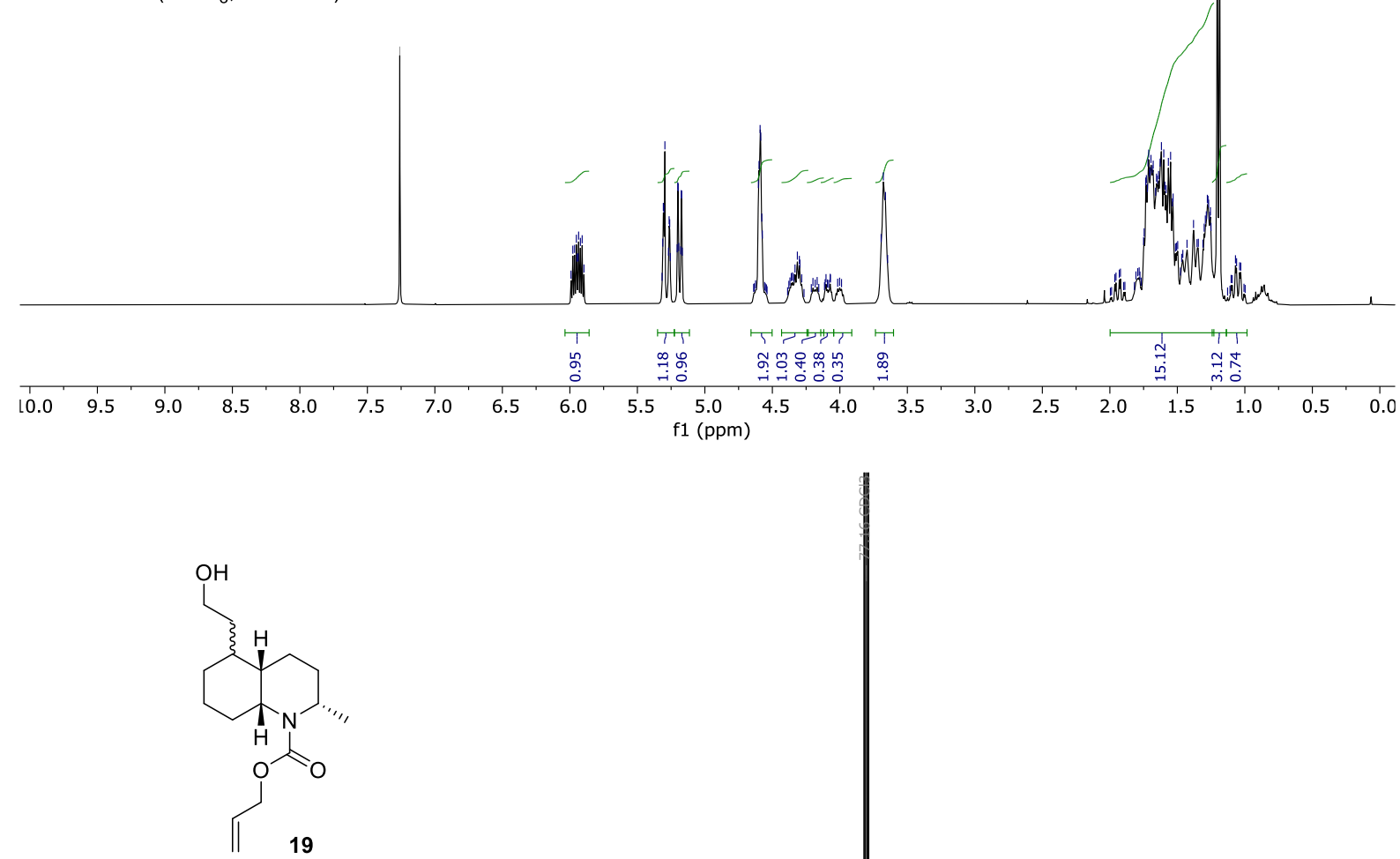

${ }^{13} \mathrm{C}\left\{{ }^{1} \mathrm{H}\right\}$ NMR $\left(\mathrm{CDCl}_{3}, 101 \mathrm{MHz}\right)$

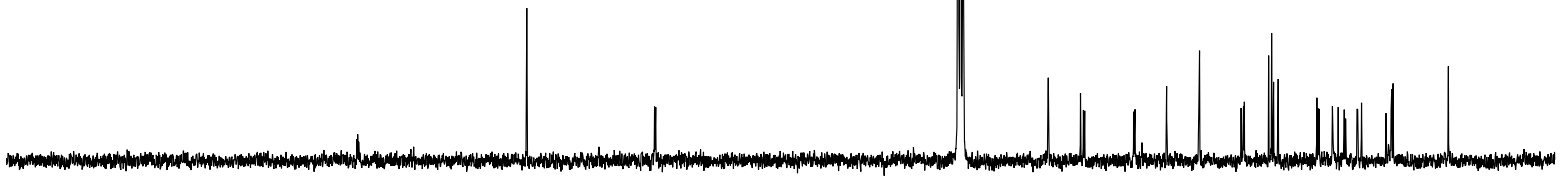

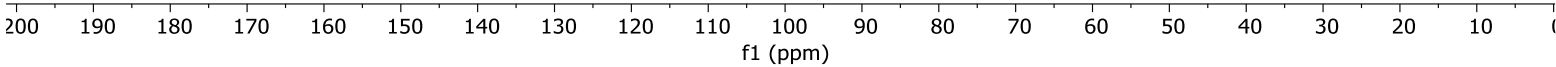




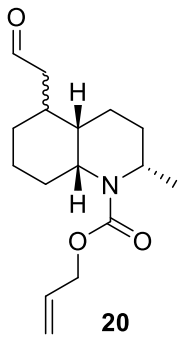

${ }^{1} \mathrm{H}$ NMR $\left(\mathrm{CDCl}_{3}, 400 \mathrm{MHz}\right)$
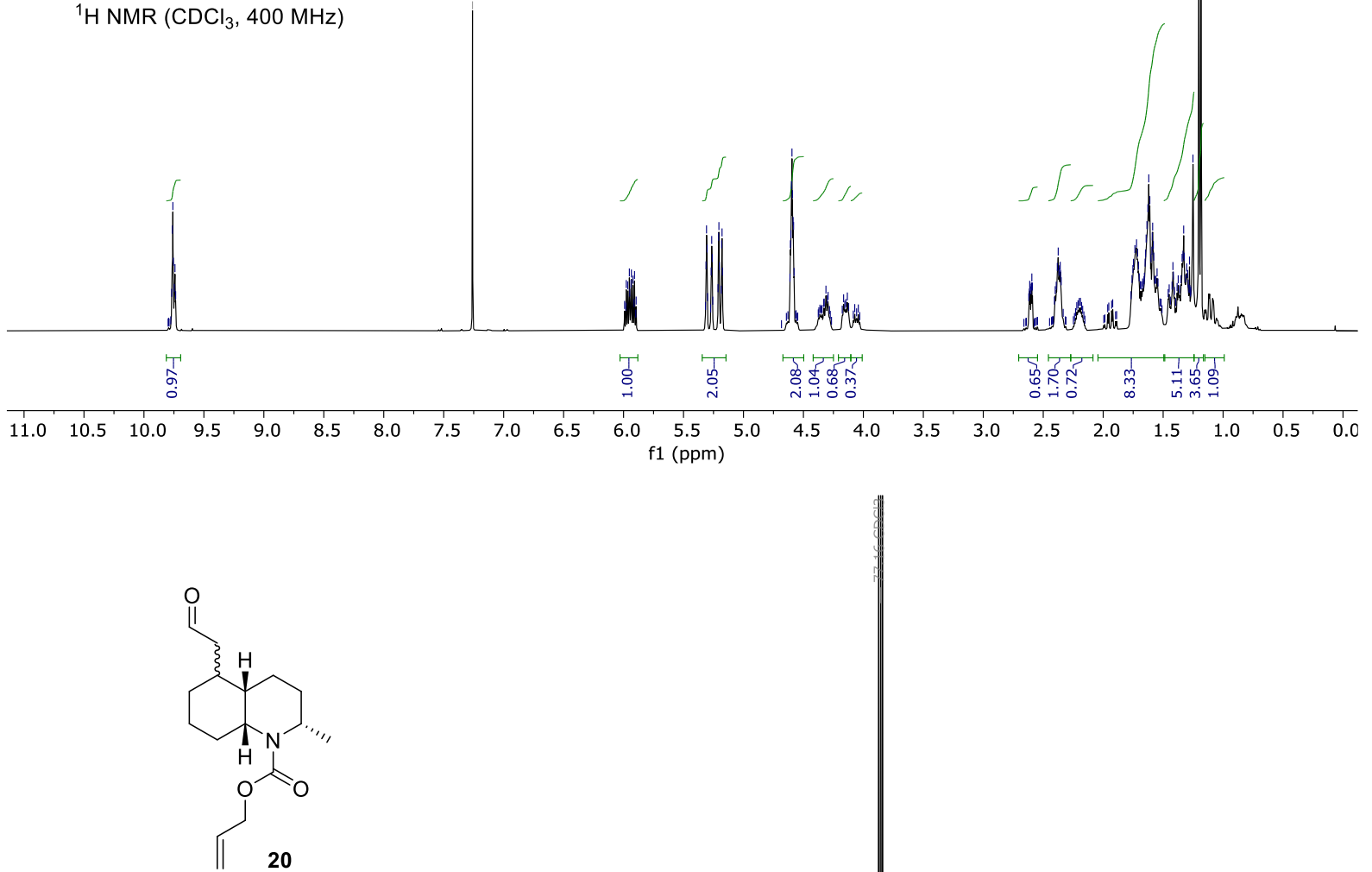

${ }^{13} \mathrm{C}\left\{{ }^{1} \mathrm{H}\right\}$ NMR $\left(\mathrm{CDCl}_{3}, 101 \mathrm{MHz}\right)$

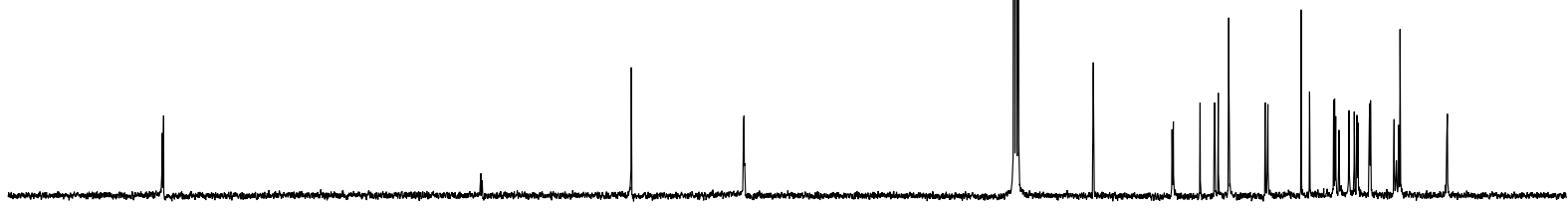

$\begin{array}{lllllllllllllllllllllll}220 & 210 & 200 & 190 & 180 & 170 & 160 & 150 & 140 & 130 & 120 & \underset{\mathrm{f} 1(\mathrm{ppm})}{110} & 100 & 90 & 80 & 70 & 60 & 50 & 40 & 30 & 20 & 10 & 0\end{array}$ 


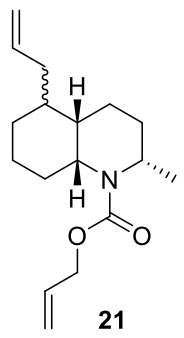

${ }^{1} \mathrm{H} \mathrm{NMR}\left(\mathrm{CDCl}_{3}, 400 \mathrm{MHz}\right)$
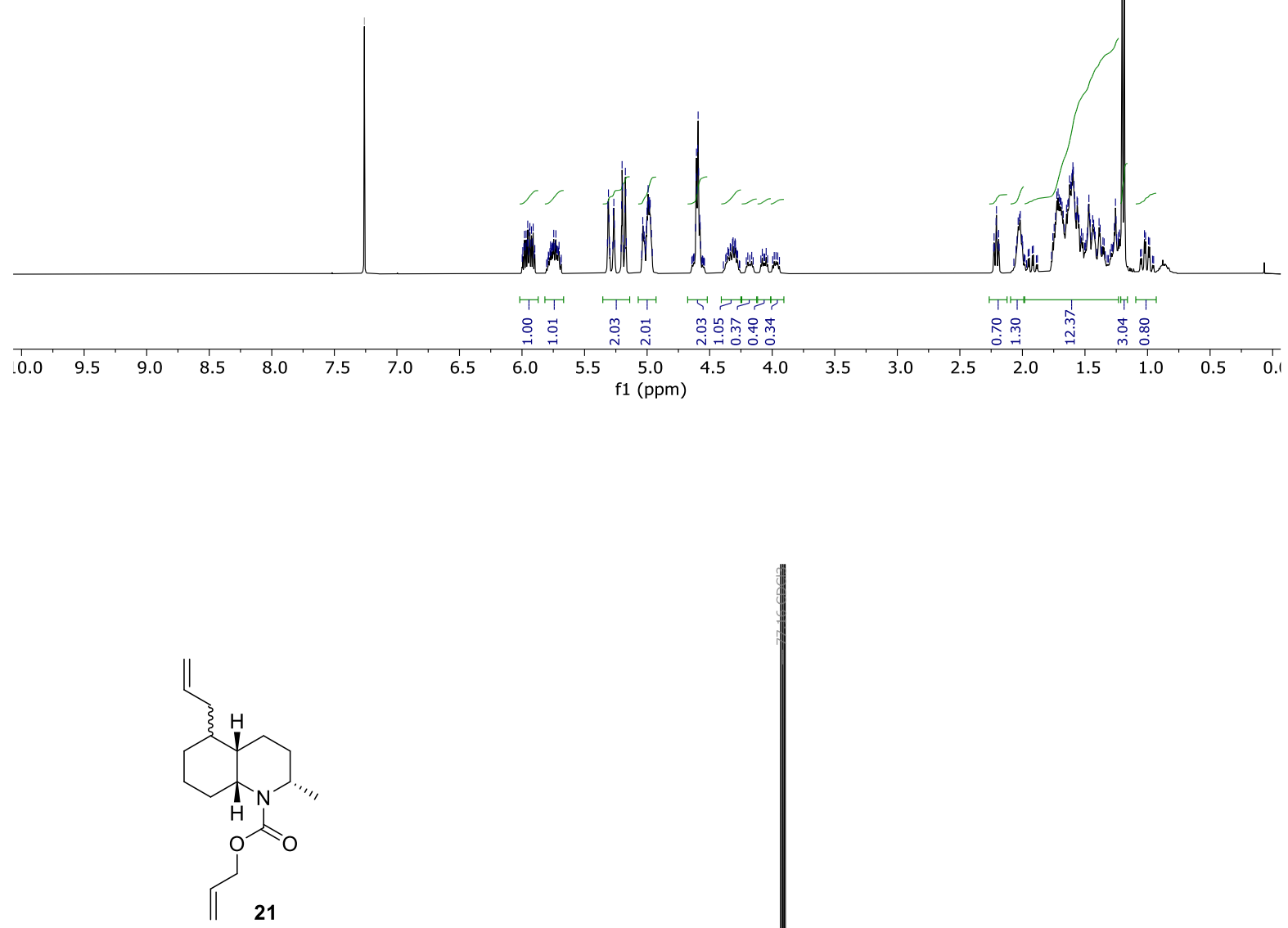

${ }^{13} \mathrm{C}\left\{{ }^{1} \mathrm{H}\right\}$ NMR $\left(\mathrm{CDCl}_{3}, 101 \mathrm{MHz}\right)$

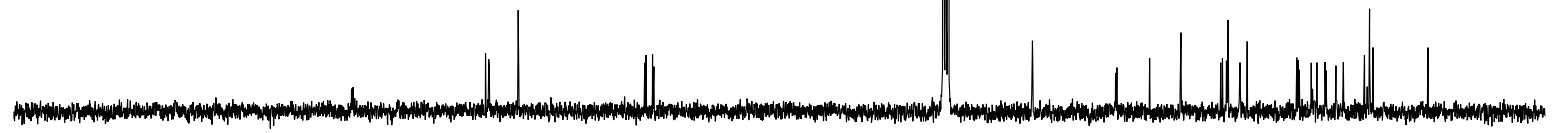

$\begin{array}{lllllllllllllllllllll}00 & 190 & 180 & 170 & 160 & 150 & 140 & 130 & 120 & 110 & \underset{\mathrm{f} 1(\mathrm{ppm})}{100} & 90 & 80 & 70 & 60 & 50 & 40 & 30 & 20 & 10 & 0\end{array}$ 
<smiles>C=CC[C@H]1CCC[C@H]2NC(C)CC[C@H]12</smiles>

22a

${ }^{1} \mathrm{H} \mathrm{NMR}\left(\mathrm{CDCl}_{3}, 400 \mathrm{MHz}\right)$
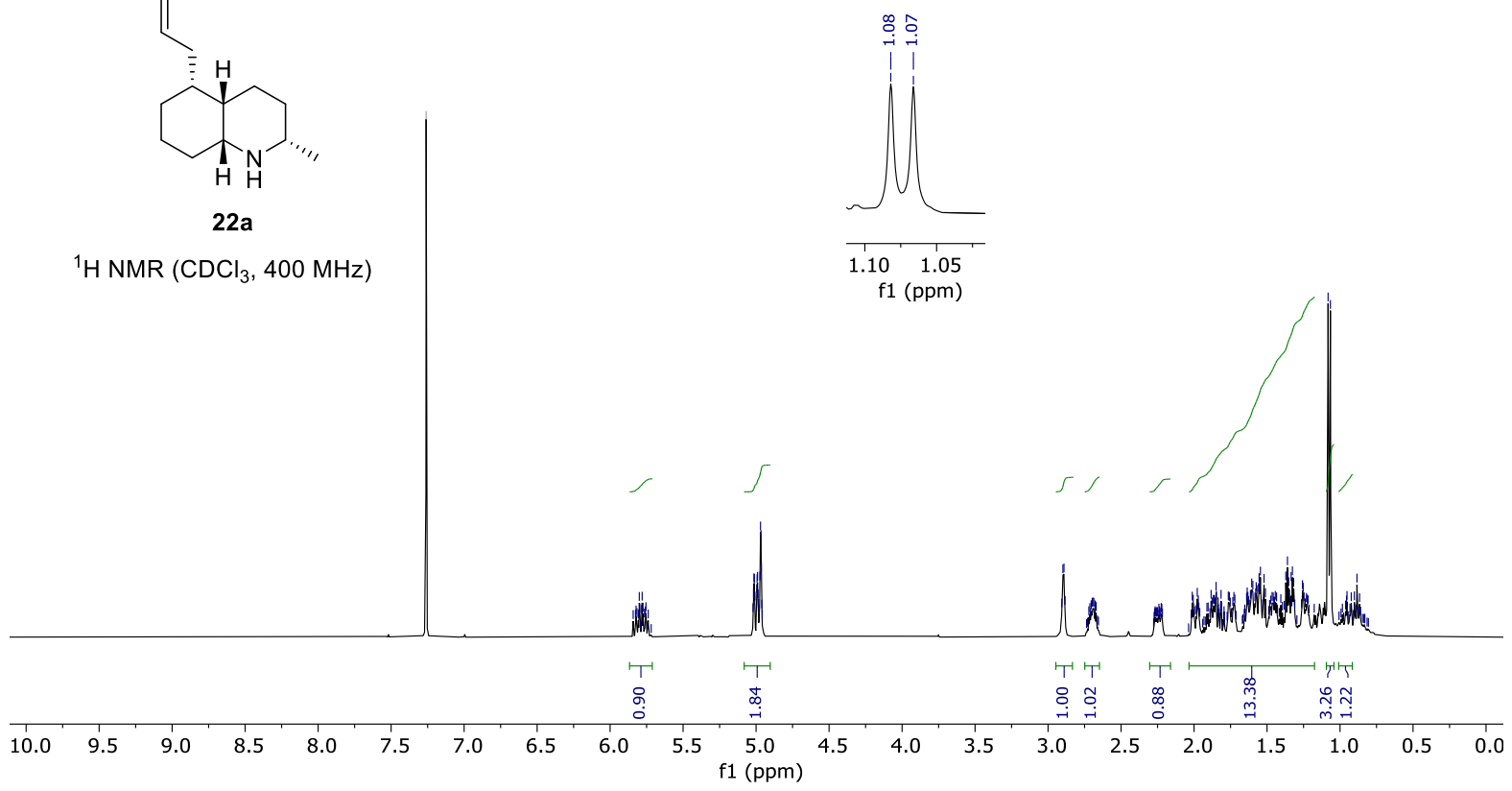

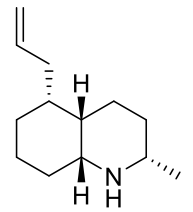

22a

${ }^{13} \mathrm{C}\left\{{ }^{1} \mathrm{H}\right\}$ NMR $\left(\mathrm{CDCl}_{3}, 101 \mathrm{MHz}\right)$

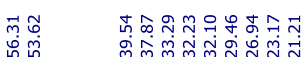

11

। पर। ?
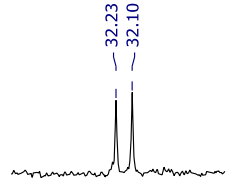

$\begin{array}{llll}33.0 & 32.5 & 32.0 & 31.5\end{array}$ f1 (ppm)

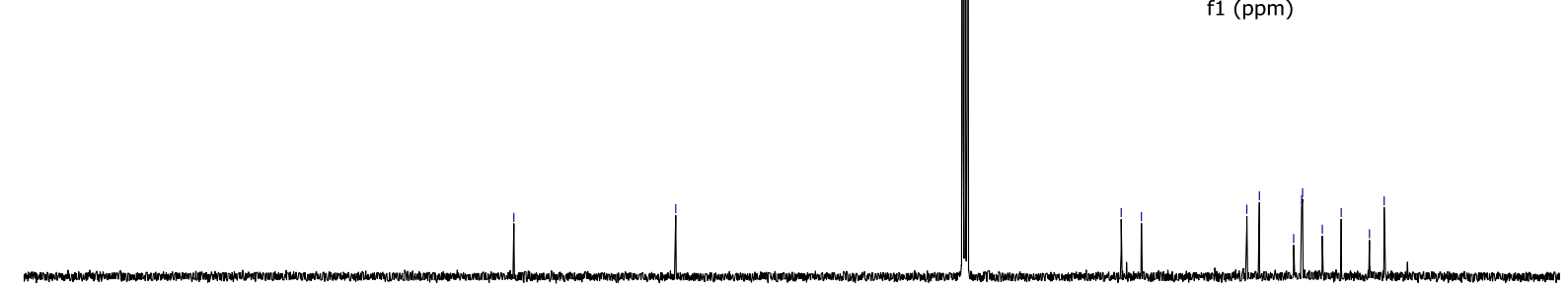

200

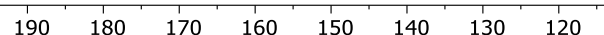

100

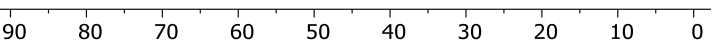




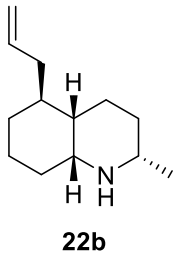

${ }^{1} \mathrm{H} \mathrm{NMR}\left(\mathrm{CDCl}_{3}, 400 \mathrm{MHz}\right)$
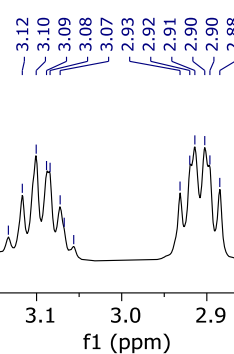

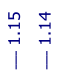
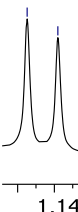

$\mathrm{f1}(\mathrm{ppm})$

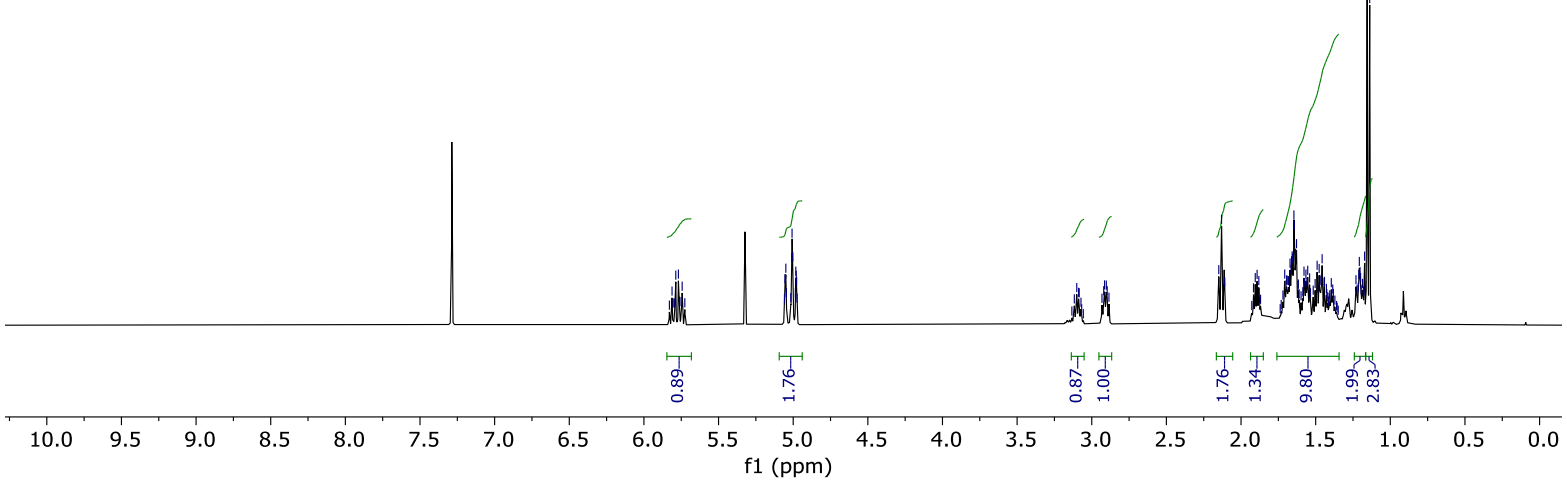

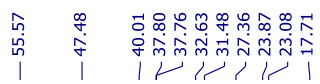

윤

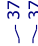

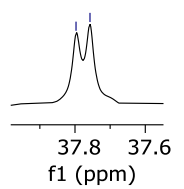

${ }^{13} \mathrm{C}\left\{{ }^{1} \mathrm{H}\right\} \operatorname{NMR}\left(\mathrm{CDCl}_{3}, 101 \mathrm{MHz}\right)$

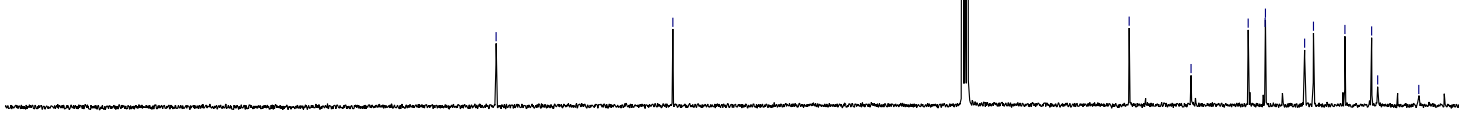

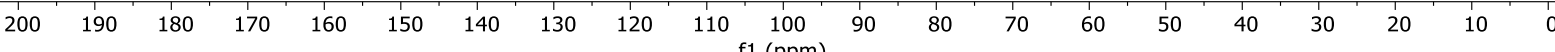




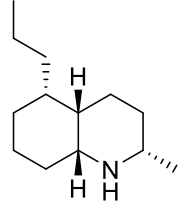

cis-195J-a

${ }^{1} \mathrm{H} \mathrm{NMR}\left(\mathrm{CDCl}_{3}, 400 \mathrm{MHz}\right)$
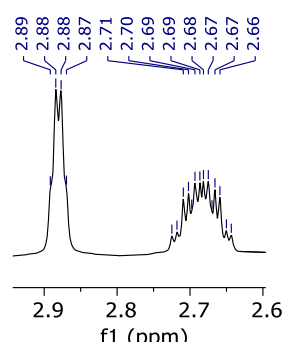

f1 (ppm)

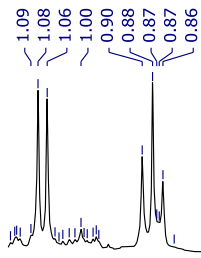

$\begin{array}{lllll}1.1 & 1.0 & 0.9 & 0.8\end{array}$ f1 (ppm)
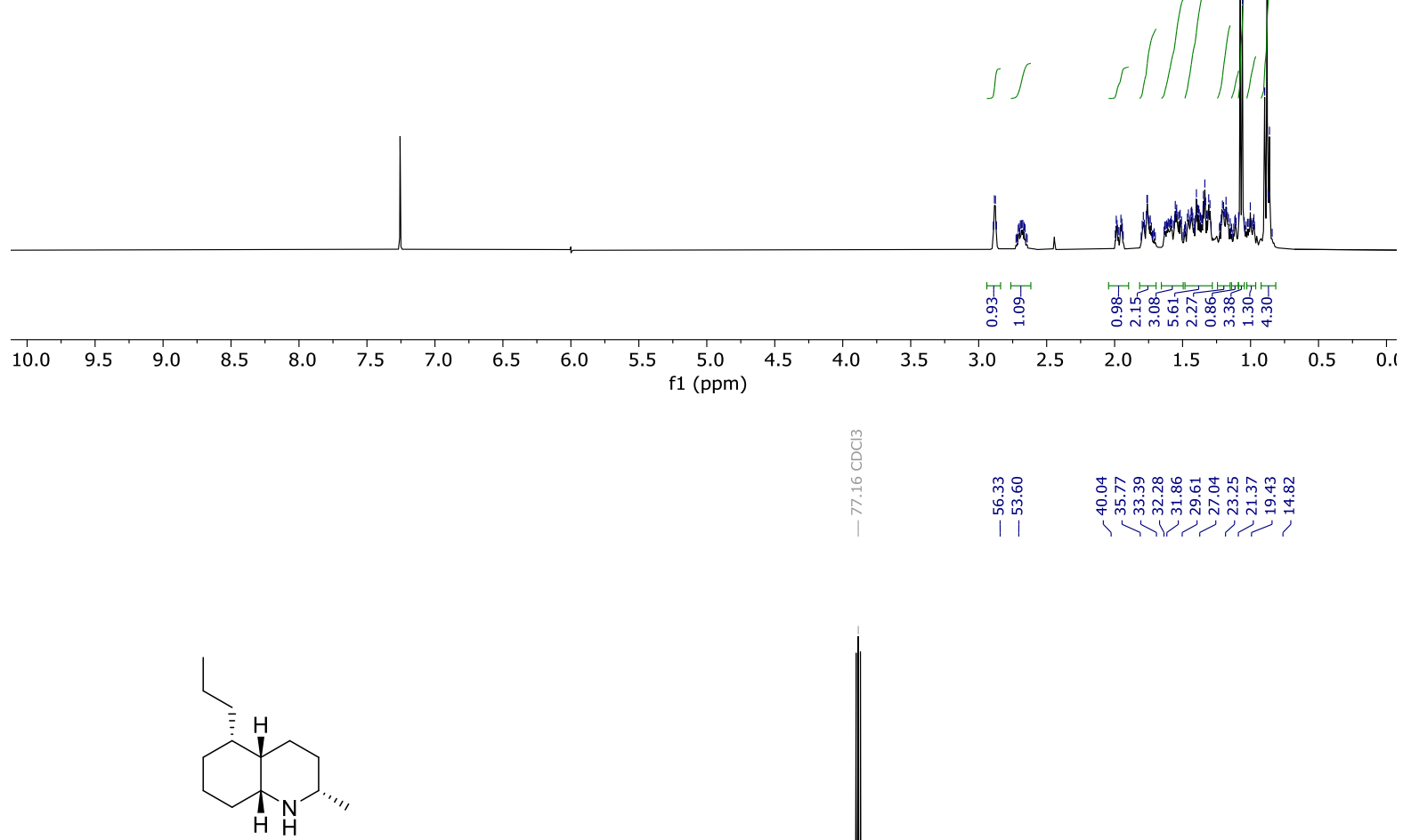

cis-195J-a

${ }^{13} \mathrm{C}\left\{{ }^{1} \mathrm{H}\right\} \operatorname{NMR}\left(\mathrm{CDCl}_{3}, 101 \mathrm{MHz}\right)$ 

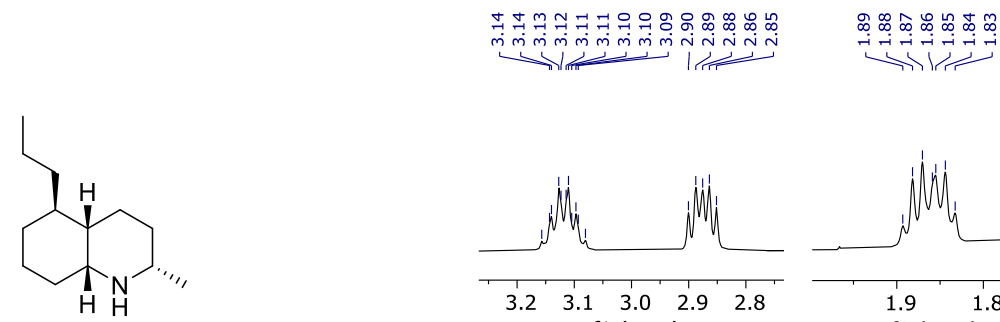

cis-195J-b

${ }^{1} \mathrm{H}$ NMR $\left(\mathrm{CDCl}_{3}, 400 \mathrm{MHz}\right)$
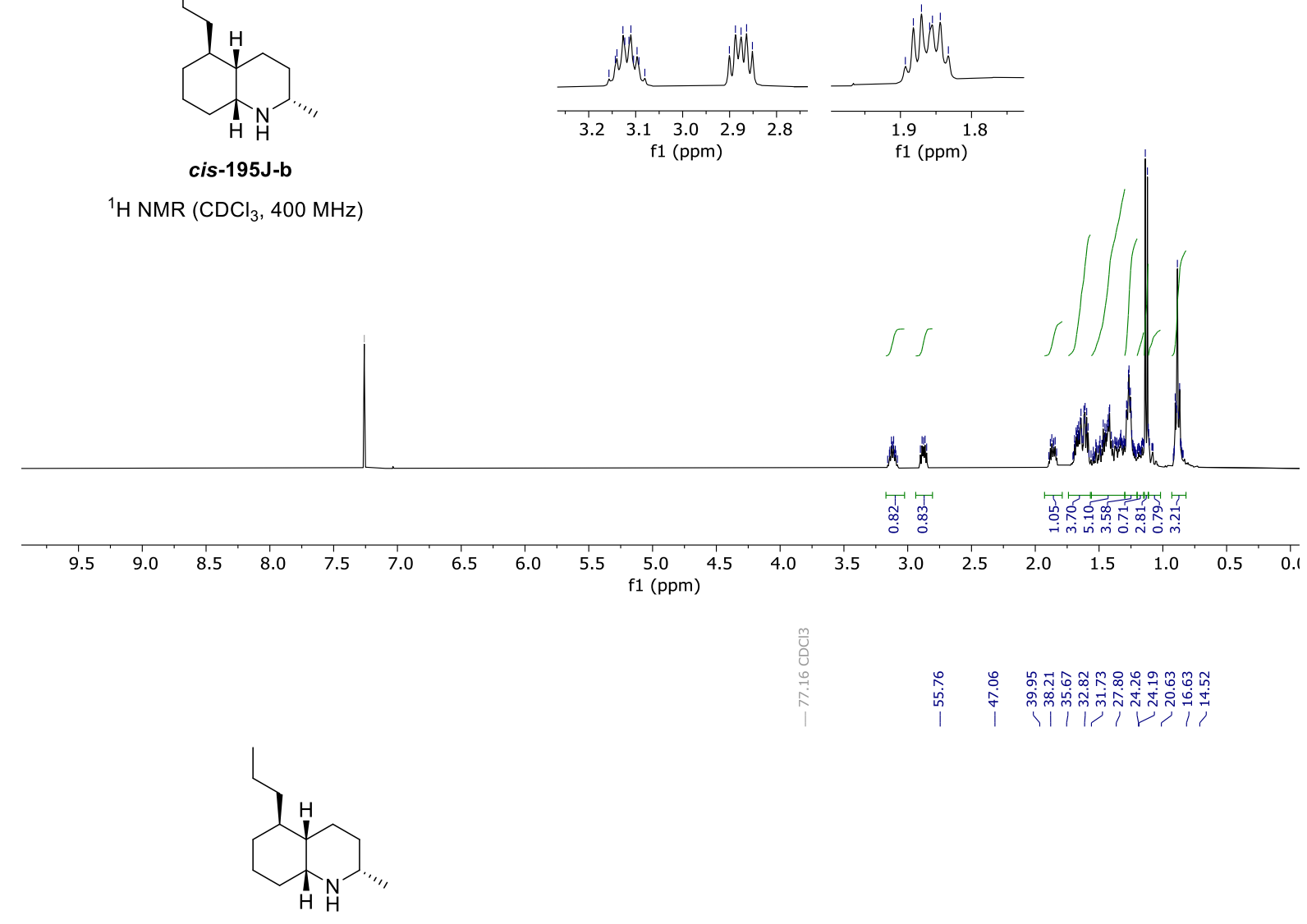

cis-195J-b

${ }^{13} \mathrm{C}\left\{{ }^{1} \mathrm{H}\right\}$ (APT) NMR $\left(\mathrm{CDCl}_{3}, 101 \mathrm{MHz}\right)$

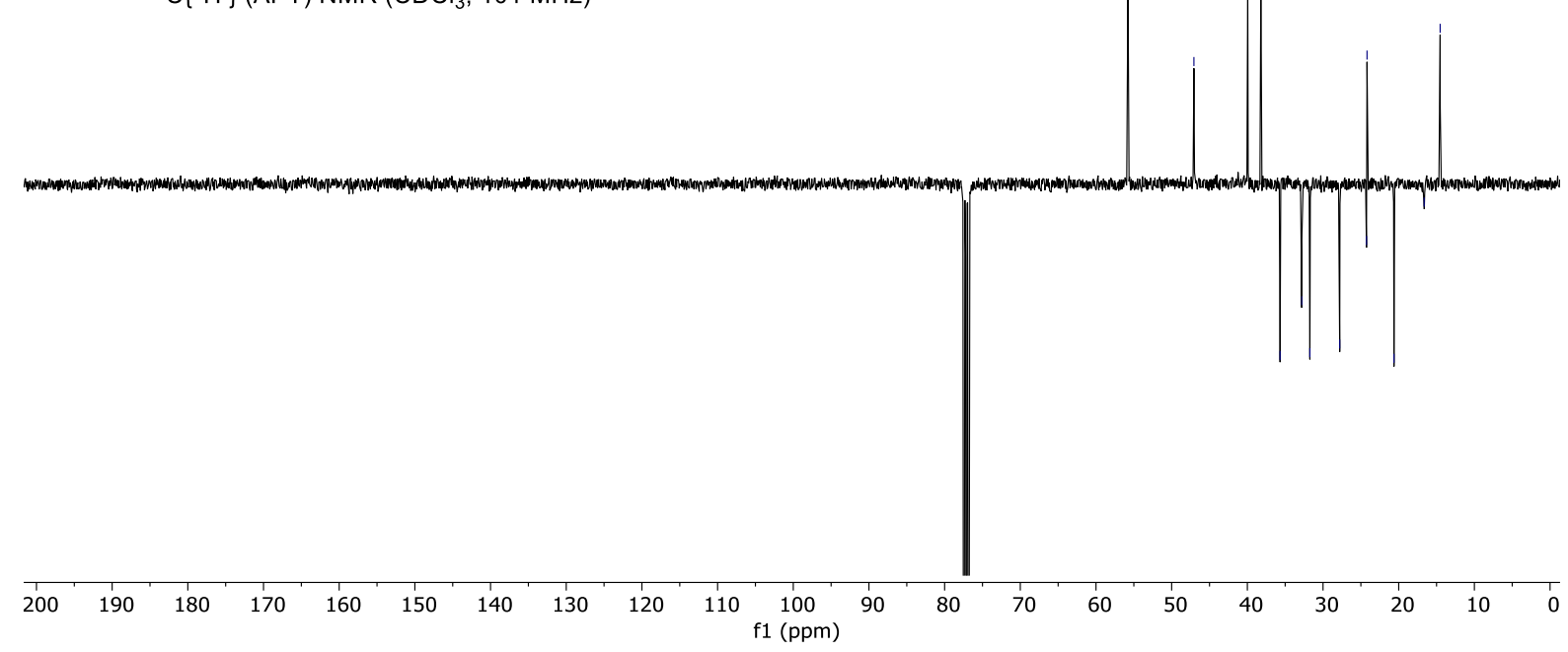



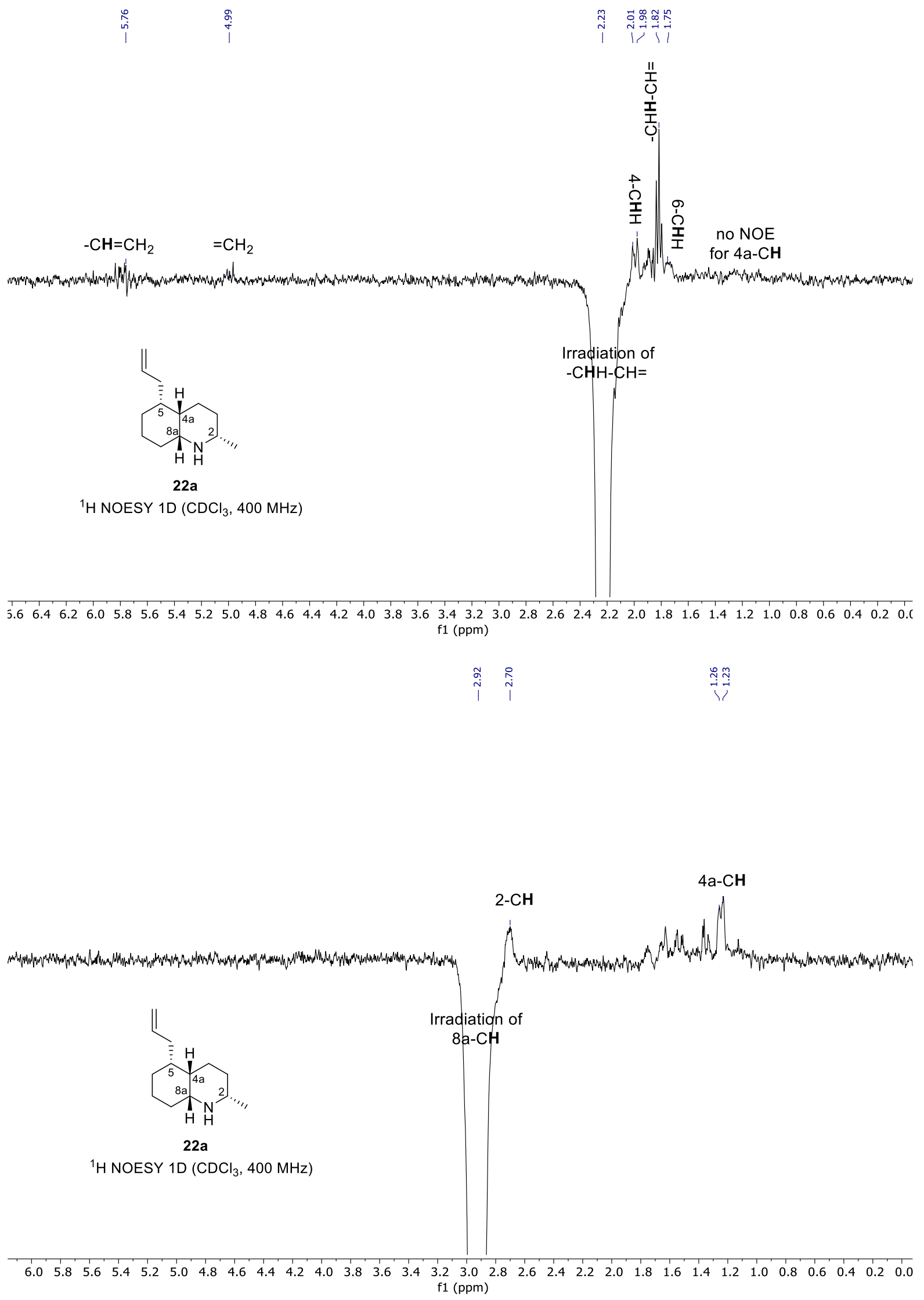

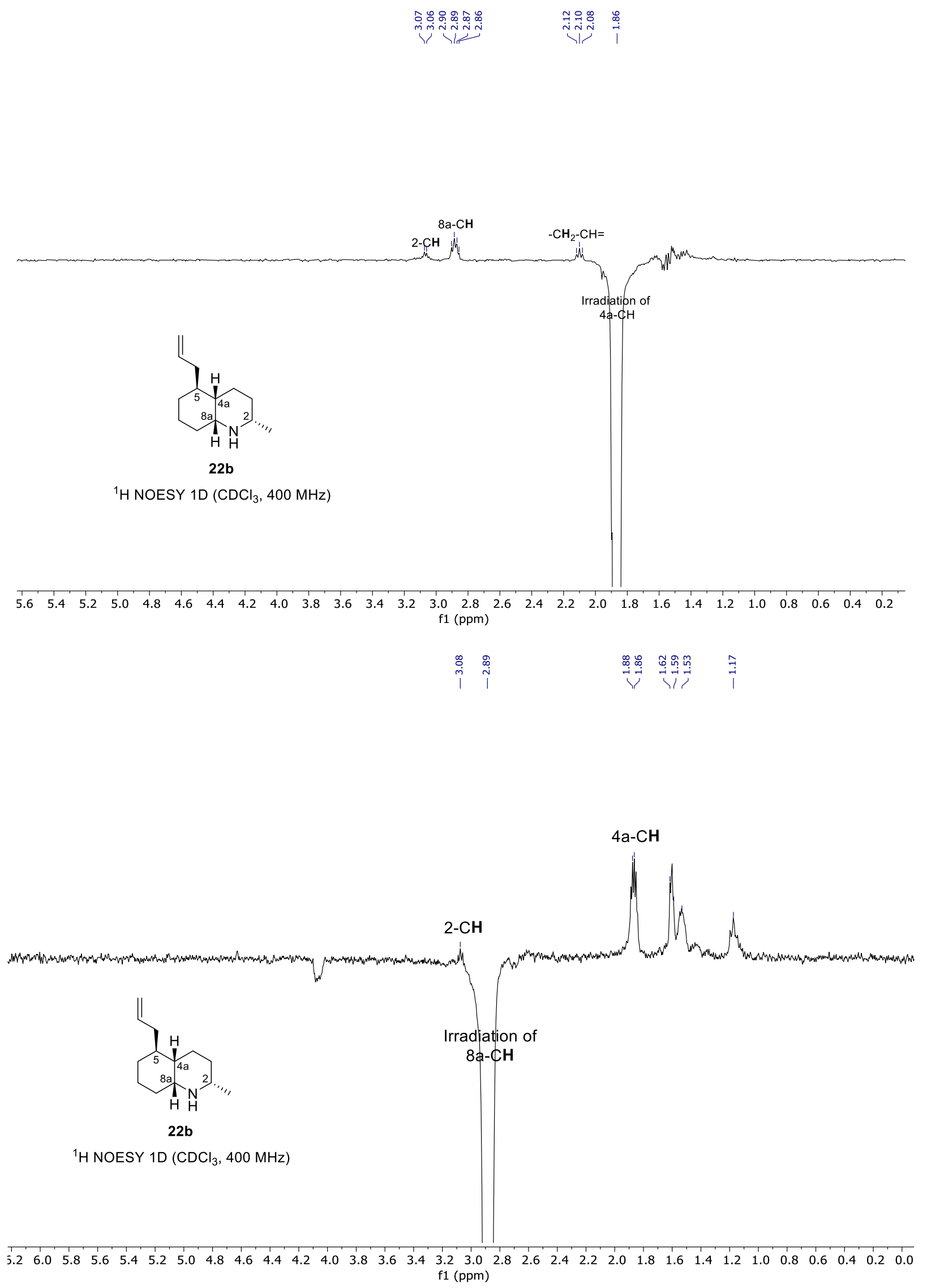


\section{Chromatogram}

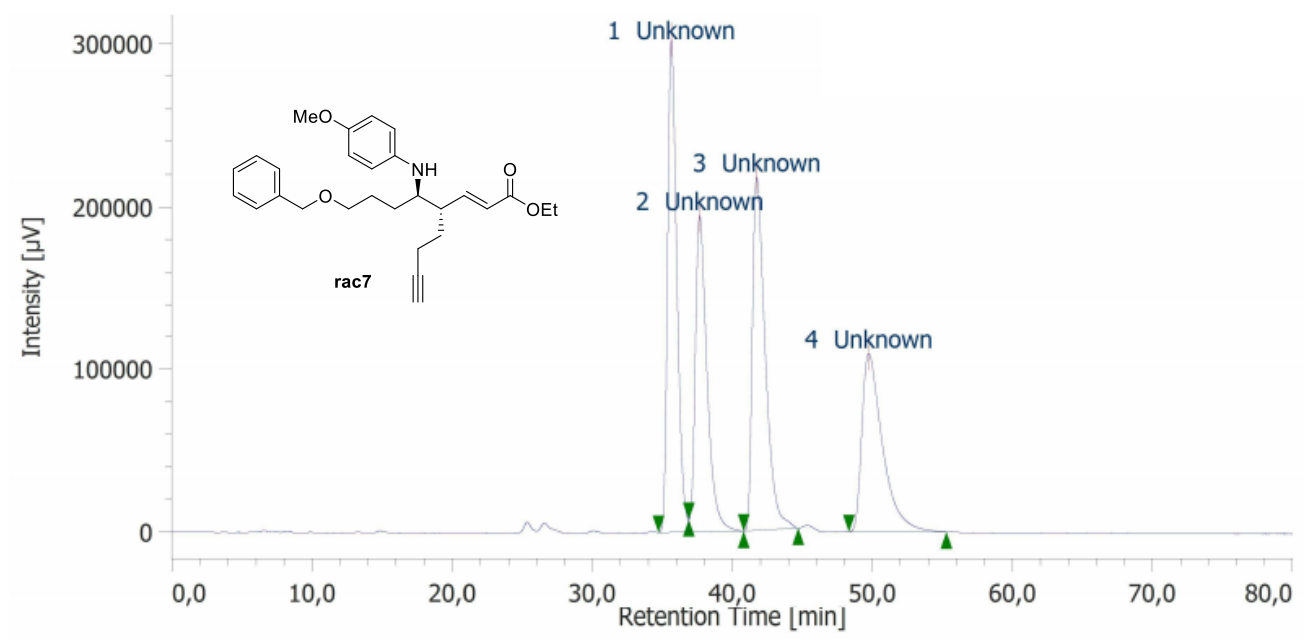

\begin{tabular}{|c|c|c|c|c|c|c|c|c|c|c|c|}
\hline \begin{tabular}{|l|l|} 
Peak Name \\
\end{tabular} & $\mathrm{CH}$ & tR [min] & Area $[\mu \mathrm{V}-\mathrm{sec}]$ & Height $[\mu \mathrm{V}]$ & Area\% & Height\% & Quantity & \begin{tabular}{|l|} 
NTP \\
\end{tabular} & Resolution & Symmetry Factor & Warning \\
\hline \begin{tabular}{|l|l|}
1 & Unknown \\
\end{tabular} & 11 & 35,627 & 13887353 & 302281 & 27,657 & 36,732 & $\mathrm{~N} / \mathrm{A}$ & 14088 & 1,524 & 1,398 & \\
\hline \begin{tabular}{|l|l|}
2 & Unknown \\
\end{tabular} & 11 & 37,650 & 11320505 & 194456 & 22,545 & 23,629 & $\mathrm{~N} / \mathrm{A}$ & 10616 & 2,652 & 543 & \\
\hline \begin{tabular}{|l|l|}
3 & Unn \\
\end{tabular} & 11 & 41,723 & 13 & 17102 & 27,476 & 26,381 & $\mathrm{~N} / \mathrm{A}$ & 10638 & 3,846 & 1,804 & \\
\hline \begin{tabular}{|l|l|}
4 & Unknown \\
\end{tabular} & 11 & 49,723 & 11207900 & 109102 & 22,321 & 13,258 & $\mathrm{~N} / \mathrm{A}$ & 6069 & $\mathrm{~N} / \mathrm{A}$ & 1,950 & \\
\hline
\end{tabular}

Chromatogram

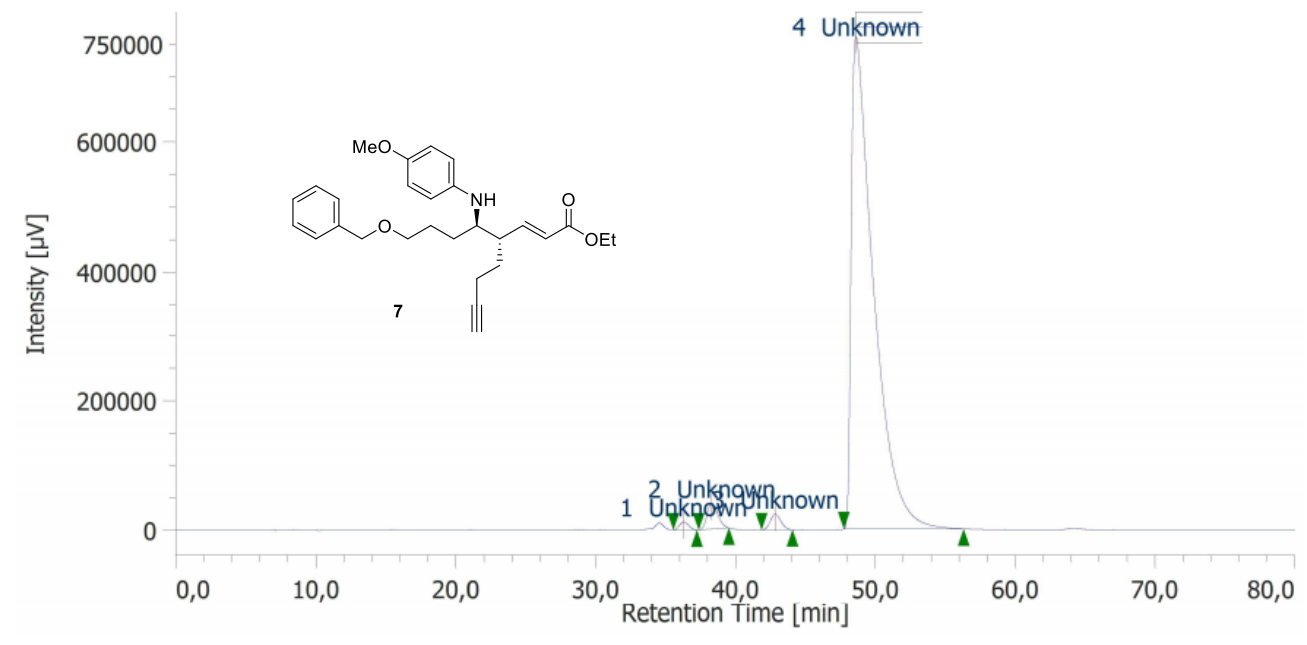

\begin{tabular}{|l|l|r|r|r|r|r|r|r|r|r|r|r|}
\hline$\#$ & Peak Name & $\mathrm{CH}$ & tR [min] & Area $[\mu \mathrm{V} \cdot \mathrm{sec}]$ & Height $[\mu \mathrm{V}]$ & Area: & Height\% & Quantity & NTP & Resolution & Symmetry Factor & Warning \\
\hline 1 & Unknown & 2 & 36,275 & 547196 & 12347 & 0,622 & 1,479 & $\mathrm{~N} / \mathrm{A}$ & 14742 & 1,536 & 1,135 & \\
\hline 2 & Unknown & 2 & 38,283 & 2126367 & 39904 & 2,417 & 4,780 & $\mathrm{~N} / \mathrm{A}$ & 11505 & 3.222 & 1,246 & \\
\hline 3 & Unknown & 2 & 42,825 & 1276778 & 24173 & 1,451 & 2,895 & $\mathrm{~N} / \mathrm{A}$ & 14990 & 2,791 & 1,131 & \\
\hline 4 & Unknown & 2 & 48,600 & 84017459 & 758482 & 95,509 & 90,846 & $\mathrm{~N} / \mathrm{A}$ & 4995 & $\mathrm{~N} / \mathrm{A}$ & 3,390 & \\
\hline
\end{tabular}




\begin{tabular}{|c|c|c|c|}
\hline \multicolumn{4}{|c|}{ NMR data of $c i s-195 J-a$ and $c i s-195 J-b$} \\
\hline \multicolumn{2}{|c|}{${ }^{\mathbf{1}} \mathbf{H} \mathbf{N M R}\left(\mathrm{CHCl}_{3}, 400 \mathrm{MHz}\right)$} & \multicolumn{2}{|c|}{${ }^{13} \mathbf{C ~ N M R}\left(\mathrm{CHCl}_{3}, 101 \mathrm{MHz}\right)$} \\
\hline cis-195J-a & $c i s-195 \mathrm{~J}-\mathrm{b}$ & $c i s-195 \mathrm{~J}-\mathbf{a}$ & cis-195J-b \\
\hline $2.88(\mathrm{q}, 1 \mathrm{H}, J=3.0 \mathrm{~Hz})$ & $3.12(\mathrm{~m}, 1 \mathrm{H})$ & 56.3 & 55.8 \\
\hline $2.68(\mathrm{dqd}, 1 \mathrm{H}, J=10.6,6.3,2.7 \mathrm{~Hz})$ & $2.88(\mathrm{dt}, 1 \mathrm{H}, J=9.7,5.1 \mathrm{~Hz})$ & 53.6 & 47.1 \\
\hline $2.01-1.93(\mathrm{~m}, 1 \mathrm{H})$ & $1.86(\mathrm{dt}, 1 \mathrm{H}, J=10.5,4.5 \mathrm{~Hz})$ & 40.0 & 39.9 \\
\hline $1.82-1.67(\mathrm{~m}, 2 \mathrm{H})$ & $1.71-1.56(\mathrm{~m}, 4 \mathrm{H})$ & 35.8 & 38.2 \\
\hline $1.65-1.50(\mathrm{~m}, 3 \mathrm{H})$ & $1.55-1.30(\mathrm{~m}, 5 \mathrm{H})$ & 33.4 & 35.7 \\
\hline $1.49-1.27(\mathrm{~m}, 5 \mathrm{H})$ & $1.29-1.20(\mathrm{~m}, 4 \mathrm{H})$ & 32.3 & 32.8 \\
\hline $1.23-1.14(\mathrm{~m}, 2 \mathrm{H})$ & $1.19-1.14(\mathrm{~m}, 1 \mathrm{H})$ & 31.9 & 31.7 \\
\hline $1.14-1.08(\mathrm{~m}, 1 \mathrm{H})$ & $1.13(\mathrm{~d}, 3 \mathrm{H}, J=6.7 \mathrm{~Hz})$ & 29.6 & 27.8 \\
\hline $1.07(\mathrm{~d}, 3 \mathrm{H}, J=6.3 \mathrm{~Hz})$ & $1.12-1.01(\mathrm{~m}, 1 \mathrm{H})$ & 27.0 & 24.3 \\
\hline $1.03-0.94(\mathrm{~m}, 1 \mathrm{H})$ & $0.89(\mathrm{t}, 3 \mathrm{H}, J=7.3 \mathrm{~Hz})$ & 23.3 & 24.2 \\
\hline $0.88(\mathrm{t}, 3 \mathrm{H}, J=7.2 \mathrm{~Hz})$ & & 21.4 & 20.6 \\
\hline $0.92-0.82(\mathrm{~m}, 1 \mathrm{H}) \mathrm{ppm}$ & & 19.4 & 16.6 \\
\hline $\begin{array}{r}\text { Optical rotation: } c i s-\mathbf{1 9 5} \mathrm{J}-\mathbf{a}[a \\
\\
\text { cis } \mathbf{- 1 9 5 J - b}[a\end{array}$ & $\begin{array}{l}=-12.4\left(c=0.45, \mathrm{CHCl}_{3}\right) \\
=+17.5\left(c=0.28, \mathrm{CHCl}_{3}\right)\end{array}$ & 14.8 & 14.5 \\
\hline
\end{tabular}

